\title{
Probiotic Foods and Supplements Interventions for Metabolic Syndromes: A Systematic Review and Meta-Analysis of Recent Clinical Trials
}

\author{
Yue Dong ${ }^{a}$ Mengjie $\mathrm{Xu}^{\mathrm{b}}$ Lanming Chen ${ }^{\mathrm{b}}$ Amir Bhochhibhoya ${ }^{\mathrm{c}}$ \\ ${ }^{a}$ Department of Anthropology, University of Oklahoma, Norman, OK, USA; ${ }^{b}$ College of Food Science and \\ Technology, Shanghai Ocean University, Shanghai, China; ' ${ }^{C}$ William Preston Turner School of Nursing, Lander \\ University, Greenwood, SC, USA
}

\section{Keywords}

Metabolic syndrome · Probiotics · Lactobacillus .

Bifidobacterium · Anthropometric change

\begin{abstract}
Background: Individual clinical trials suggested that when treated with probiotic foods or supplements with Lactobacillus and Bifidobacterium, specific symptoms of metabolic syndrome (MetS) could be alleviated, but the results have been inconclusive. Aims: The objective of the present meta-analysis was to use anthropometric and biochemical as indicators to evaluate the efficacy of using these probiotic foods or supplements among individuals with MetS. Methods: PubMed, Cochrane Library, and CINAHL Plus were used to collect randomized controlled trials (RCTs) studies published from January 2000 to January 2018. Studies were included if they had at least one of the following outcome measurements: body mass index (BMI), waist circumference, hip circumference, waist-to-hip ratio, body fat mass (BFM), body fat percentage (BFP), systolic blood pressure (SBP), diastolic blood pressure (DBP), fasting glucose, fasting insulin, total cholesterol (TC), high-density lipoprotein cholesterol (HDL-
\end{abstract}

\section{KARGER}

(c) 2019 S. Karger AG, Basel

E-Mail karger@karger.com

www.karger.com/anm
C), low-density lipoprotein cholesterol (LDL-C), triglycerides, and/or hemoglobin A1c (HbA1c). Results: The 356 records were identified during the literature search, of which only 18 met the selection criteria. The 18 RCTs with a total of 1,544 participants were included in this analysis. This meta-analysis indicated that there were no significant differences of $\mathrm{BMI}, \mathrm{BFM}$, waist circumference, hip circumference, waist-tohip ratio, SBP, DBP, fasting glucose, fasting insulin, $T C, \mathrm{HDL}-$ $\mathrm{C}, \mathrm{HbA} 1 \mathrm{c}$, or triglycerides between the intervention and control groups. Significant standardized mean net differences were found in the BFP and LDL-C between the intervention and control groups. Conclusions: The results indicated that probiotic food and supplement with Lactobacillus and Bifidobacterium could be used as interventions to improve specific anthropometric and biochemical outcomes among individuals with MetS. However, probiotic treatment alone could not reduce overall health risks. In addition, there were methodological drawbacks among reviewed studies, and further research is needed.

(c) 2019 S. Karger AG, Basel

Ms. Mengjie Xu and Yue Dong contributed equally to this work.
Yue Dong

Department of Anthropology

University of Oklahoma, 455 West Lindsey

Dale Hall Tower 521, Norman, OK 73019 (USA)

E-Mail Yue.Dong-1@ou.edu 


\section{Introduction}

According to the American Heart Association/National Heart, Lung, and Blood Institute, metabolic syndrome (MetS) is the failure of normal body metabolism functions that are related to abnormalities of serum triglyceride, lipoprotein, and cholesterol levels [1]. Several studies suggest that MetS is a cluster of physiological, biochemical, and metabolic risk conditions, which are often related to cardiovascular diseases, type 2 diabetes mellitus, and obesity [2-4]. In 2016, more than 650 million individuals were obese, and more than 1.9 billion adults were overweight [5]. In the United States, $39.8 \%$ in adults and $18.5 \%$ in youth were obese [6]. Obesity is associated with severe health risks such as cardiovascular diseases and diabetes [7]. In 2015, cardiovascular disease was the first leading cause of death (258.9 death per 100,000), and diabetes mellitus is the seventh leading cause of death in the United States (24.7 death per 100,000) [8]. Previous studies suggested that waist and hip circumferences, body mass index (BMI), blood pressure, and blood glucose could frequently be used to measure individuals' risk of developing MetS $[2,9]$. Research showed that $10^{14}$ microorganisms consisted of more than 50 bacterial phyla live in the human gastrointestinal tract $[10,11]$. Previous studies have suggested that gut microbiota play a vital role in the regulation of body metabolism function such as decomposition of carbohydrates, proteins, and lipids, absorption of both energy and nutrients, as well as gut motility [12-15]. Recent studies have suggested that MetS was associated with specific abnormalities in gut microbiota [16-18]. Food intake has a significant impact on the formation, composition, and functions of the human digestive tract microbiota [19]. By introducing microorganisms into the digestive tract, it is possible to change gut microbiota and may lead to beneficial changes in the gastrointestinal environment [20]. Recent clinical research has shown that in the novel treatment of MetS, the use of probiotic foods or supplements might help patients who have MetS to alleviate the severity of symptoms and that probiotics could change both anthropometric and biochemical outcomes of the individuals [21,22]. Le Barz et al. [23] suggested that probiotics could be used as a complementary treatment for individuals with metabolic-related problems.

Among probiotic foods or supplements, the most common strains such as Lactobacillus rhamnosus, Lactobacillus acidophilus, Lactobacillus plantarum, Lactobacillus reuteri, Lactobacillus gasseri, Lactobacillus paracasei, Lactobacillus casei, Lactobacillus salivarius, Bifidobacterium animalis subsp. Lactis, and Bifidobacterium bifidum are affiliated with the Lactobacillus (L.) and Bifidobacterium (B.) genera [24-26]. Previous studies have linked these 2 genera with human gut health and metabolic functions [27, 28]. Lactobacillus and Bifidobacterium genera are the predominant genera in the intestinal lumina, and Lactobacillus is predominant in the mucosaassociated surface [29].

Furthermore, studies show that in recent years, news outlets, commercial websites, and noncommercial websites have made more claims about the beneficial medical effect of using probiotic foods or supplements for treating MetS [30, 31]. Nevertheless, to date, no meta-analysis has been conducted to study the efficacy of using Lactobacillus and Bifidobacterium foods or supplements to alleviate the symptoms of MetS. Thus, it is crucial to systematically review the existing evidence regarding the using of probiotics in treating MetS. This study was the first to investigate the effect of Lactobacillus and Bifidobacterium on MetS; the authors conducted the meta-analysis with a focus on the changes of several key MetS anthropometric measurements and biochemical indicators.

\section{Materials and Methods}

Data Sources and Searches

The review followed the recommendations of the Preferred Reporting Items for Systematic Reviews and Meta-Analyses statement [32]. The protocol of this systematic review and meta-analysis is registered at PROSPERO (International prospective register of systematic reviews), and the registration number is CRD42018086937. Three databases PubMed, Cochrane Library, and CINAHL Plus were used to collect articles for this review. The publications were retrieved from January 2000 to January 2018. The search terms used were as follows: "Lactobacillus," "Bifidobacterium," "metabolic syndrome," "metabolic dysfunction," "obesity," "cultured milk products," and "probiotics." Also, "weight loss" and "weight control" were later added to the search term sets.

The inclusion criteria of this article were as follows: studies of randomized controlled trials (RCTs) that compared the effects of using probiotic foods or supplements (regardless of strains, forms, dosages, and duration of treatments) with controls in treating MetS. Furthermore, all included studies needed to have at least one of the following anthropometric or biochemical indicators: BMI, body fat mass (BFM), body fat percentage (BFP), waist circumference, hip circumference, waist-to-hip ratio, systolic blood pressure $(\mathrm{SBP})$, diastolic blood pressure (DBP), fasting glucose, fasting insulin, total cholesterol (TC), high-density lipoprotein cholesterol (HDL-C), low-density lipoprotein cholesterol (LDL-C), hemoglobin A1c (HbA1c), or triglycerides. This article included studies with 2 or more treatment arms.

The exclusion criteria of this review were as follows: research subjects were nonhuman; there were no intervention or prevention program being evaluated; studies did not have RCTs as research design (either a cohort study, a case-control study, cross- 


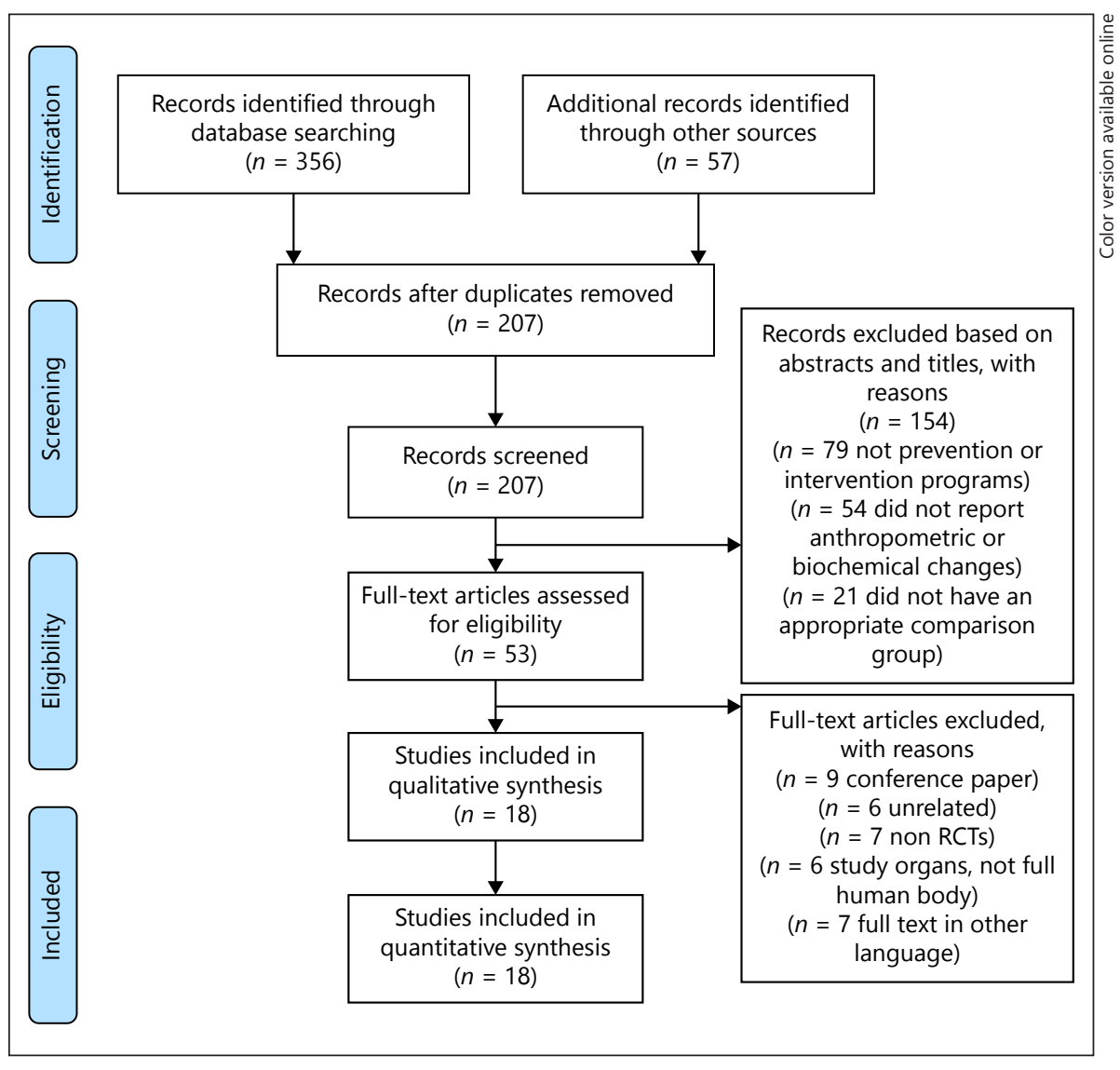

Fig. 1. PRISMA 2009 flow diagram.

sectional study, a review, or a commentary article); studies did not have the aforementioned outcome/measurements; or publications were not in English.

The initial search yielded 356 articles, and 57 additional records were identified through Google Scholar. After removing duplicates, 207 records were examined. Based on the information from the titles and abstracts, the authors removed 154 articles because for the following reasons: they were not intervention or prevention programs $(n=79)$, or did not report anthropometric or biochemical changes $(n=54)$, or did not have an appropriate comparison group $(n=21)$. The remaining 53 articles were assessed for eligibility. After full-text examination, 35 articles were excluded from the meta-analysis for the following reasons: conference paper $(n=9)$, unrelated $(n=6)$, non-RCTs $(n=7)$, only study of organs not full human body $(n=6)$, full text in other languages than English $(n=$ 7 ). Thus, 18 articles were included in the qualitative synthesis and quantitative analyses (meta-analysis; Fig. 1).

The selected studies were checked and reviewed independently by 4 researchers. Two of them have expertise in evidence-based medicine, and the other 2 researchers are experts in food microbiology. Three researchers independently conducted the study selection and abstraction of data. Any disagreement about the search strategies among the investigators in data selection was resolved through a panel discussion with the fourth researcher. The following information about eligible articles was collected: study design, duration of treatment, sample size, characteristics of research subjects, dosage, strains of the bacterium, the form of intervention (food, capsules or pills), and changes of anthropometric and biochemical indicators. The full text of all studies was assessed for eligibility against the inclusion criteria.

\section{Data Extraction and Risk of Bias Assessment}

Data were extracted on the allocation, endpoint classification, intervention model, masking, primary purpose of the clinical trial, condition, sample size, age, inclusion criteria of the participants, description of treatment arms, per-day dosage, form, duration, strain of bacterium, anthropometric indicators, biochemical indicators, level of evidence, and risk of bias. Based on the recommendations that were outlined in the Cochrane Handbook, the authors conducted assessments of the risk of bias regarding the following aspect: random sequence generation, allocation concealment, blinding of participants, blinding of research personnel, blinding of outcome assessment, attrition biases, and selective reporting [33].

\section{Measurement of Outcomes}

To evaluate the results of the intervention, the authors evaluated the differences in both anthropometric and biochemical indicators in the form of standardized mean net changes. The anthropometric indicators include BMI, waist circumference, hip circumference, waist-to-hip ratio, BFM, BFP, and SBP and DBP. The biochemical indicators include fasting glucose level, fasting insulin level, lipid profile (TC level, HDL-C level, LDL-C level, triglycerides level), and HbAlc. 
Qualitative Descriptive Analysis

This meta-analysis provided descriptive analyses of the following perspectives of the reviewed studies: blinding, characteristics of participants, and intervention methods.

\section{Quantitative Statistical Analysis}

Mean net change was used to generate results for this metaanalysis with continuous variables. Mean net changes were calculated as the differences of the changes (mean value at endpoint minus mean value at baseline) for both the experimental group and the control group. SE and CI were converted to the SD for further analyses. Regarding heterogeneity, the authors used the fixed-effect model when $\mathrm{I}^{2}$ statistic $<50 \%$, and random-effect model when $\mathrm{I}^{2}$ statistic $\geq 50 \%$. As it was suggested by Higgins [34] and Schroll et al. [35], the use of $50 \%$ is rather arbitrary; however, in the published meta-analyses, values greater than $50 \%$ are generally considered substantial heterogeneity. For studies that had multiple treatment arms, the authors followed the recommendation in Cochrane Handbook (Section 7.7.3.8, Table 7.7.a), and calculated the means, the SDs, the sample sizes of each, and then combined them into a single experimental group and a single control group for comparison [33]. Since individual meta-analysis measures the same anthropometric or biochemical indicators, the author followed the recommendation in the Cochrane Handbook (Section 9.2.3.2) and calculated the standardized mean differences for each sub meta-analysis. This methodological change also allowed the authors to provide accurate effective sizes for different interventions [33]. All analyses were performed with Review Manager 5.3 [36]. In addition, the fixed and random effects models were weighted by the inverse variance. A $p$ value $<0.05$ was considered statistically significant.

\section{Results}

\section{Overall View}

Eighteen RCT studies have met the selection criteria and were reviewed and analyzed. Tables $1-3$ present synthesized data extracted from each article in this review. Table 1 shows the following information: study design, program duration, description of the participants which include the number of participants upon completion, age, and health condition; Table 2 shows the descriptions of treatment and control groups (number of participants in each group, daily dosage, strain of microbes, and form of delivery). Table 3 shows the following information regarding the anthropometric and the biochemical changes in reviewed articles.

\section{Study Design}

All reviewed articles used randomized allocations, which reduced the risk of bias, and they all employed surrogate endpoints, which provided an easy and fast outcome measurement [37]. These studies were all targeted at providing treatments for participants with symptoms

Probiotics and MetS, A Meta-Analysis of MetS. Since all reviewed studies have similarities in allocation (randomized), endpoint classification (surrogate), and primary purpose (treatment), the authors in this study did not list this information in the study design section in Table 1. As it was shown in Table 1, only the study by Brahe et al. [38] used single-blinding methods; all other studies used double-blinding in their research $(n=17)$. Ten studies used a 2 -treatment-arm design, 5 studies used a 3-treatment-arm design, 2 studies used a 4-treatment-arm design, and one study used a 5-treatment-arm design. According to Cohen et al. [39], by adding an additional treatment arm, researchers can incorporate and evaluate new treatments and reduce the time and cost for determining optimal therapies; however, an additional treatment arm does not always produce critical statistical considerations and could lead to uninterpretable or invalid outcomes.

\section{Level of Evidence and Risk of Bias}

Higgins and Green [33] and O'Neil et al. [40] proposed that different research designs could affect the strength of the body of evidence, and they proposed that double-blinded RCTs usually have the highest strength, while single-blinded RCT has moderate strength. As mentioned above, only one study [38] used a singleblind method, and all the others used double-blind methods. Also, all reviewed RCTs had small intervals. Based on the criteria from the Centre for Evidence-Based Medicine [41], these individual RCTs (with narrow CI) had a $1 \mathrm{~B}$ level of evidence. In the 18 reviewed RCTs, the durations of Sharafedtinov et al. [42] (3 weeks) and Lindsay et al. [43] (4 weeks) were below Cochrane recommended duration (6 weeks, Section 8.6, Fig. 8.6.a), which may lead to short-term outcome and attrition bias [33].

\section{Participants}

The total participants $(n=1,544)$ include individuals with large visceral fat areas $(n=210)$, individuals with obesity and hypertension ( $n=40$ with arterial hypertonia, $n=53$ without arterial hypertonia, 93 in total), individuals with hypercholesterolemia $(n=66)$, individuals with abnormally high BMI (BMI $>25.0 ; n=792)$, individuals with obesity and fatty liver $(n=20)$, at-risk pregnant and postpartum individuals $(n=323)$, and individuals with Type 2 Diabetes $(n=40$; Table 1$)$.

\section{Intervention and Control}

The participants in the intervention group were given probiotics in the form of foods, pills, or capsules. In 
Table 1. Study design, duration, participants description

\begin{tabular}{|c|c|c|c|c|c|c|}
\hline No. & Author, year & $\begin{array}{l}\text { Study } \\
\text { design }\end{array}$ & Duration & $\begin{array}{l}\text { Participants description } \\
\text { ( } n=\text { number upon completion) }\end{array}$ & Completion, $n$ & Age \\
\hline 1 & $\begin{array}{l}\text { Kadooka } \\
\text { et al. [51], } 2013\end{array}$ & 3A; DBSI & 12 weeks & Large visceral fat areas $\left(80 \cdot 2-187 \cdot 8 \mathrm{~cm}^{2}\right)$ & 210 & $35-60$ \\
\hline 2 & $\begin{array}{l}\text { Sharafedtinov } \\
\text { et al. [42], } 2013\end{array}$ & 2A; DBSI & 3 weeks & Arterial hypertonia (>130/85 mm Hg) & 40 & $30-69$ \\
\hline 3 & $\begin{array}{l}\text { Rerksuppaphol and } \\
\text { Rerksuppaphol [48], } 2015\end{array}$ & 2A; DBSI & 6 weeks & $\begin{array}{l}\text { Hypercholesterolemia (total cholesterol level } \\
\geq 200 \mathrm{mg} / \mathrm{dL} \text { W/O lipid-lowering } \\
\text { medical treatment) }\end{array}$ & 66 & Adult \\
\hline 4 & Brahe et al. [38], 2015 & $3 \mathrm{~A} ; \mathrm{SBS}$ & 6 weeks & $\begin{array}{l}\text { Women, } 1 \text { year since last menstruation, } \\
\text { no gastrointestinal diseases or chronic } \\
\text { diseases, W/O high intake of antibiotics, } \\
\text { pro- or prebiotics or fermented food }\end{array}$ & 53 & $40-70$ \\
\hline 5 & $\begin{array}{l}\text { Agerholm-Larsen } \\
\text { et al. [61], } 2000\end{array}$ & $5 \mathrm{~A} ; \mathrm{DBSI}$ & 8 weeks & $\begin{array}{l}\text { Normal BP and high BMI } 25-37.5 \text {, } \\
\text { no special diet, normal alcohol consumption, } \\
\text { no elite athletes }\end{array}$ & 70 & $18-55$ \\
\hline 6 & Sanchez et al. [57], 2013 & 2A; DBSI & 24 weeks & $\begin{array}{l}\text { Not pregnant, breastfeeding or menopausal, } \\
\text { BMI } 29-41 \text {, W/O complications }\end{array}$ & 125 & $18-55$ \\
\hline 7 & Vajro et al. [49], 2011 & 2A; DBSI & 8 weeks & $\begin{array}{l}\text { BMI }>95 \text { th, and persisting } \\
\text { hypertransaminasemia and bright liver }\end{array}$ & 20 & Children \\
\hline 8 & Ilmonen et al. [59], 2011 & $\begin{array}{l}3 \mathrm{~A} ; \mathrm{SBS} \\
\text { and DBSI }\end{array}$ & $\begin{array}{l}\text { Until the end of } \\
\text { exclusive } \\
\text { breastfeeding } \\
\text { for up to } \\
6 \text { months }\end{array}$ & $\begin{array}{l}\text { At the first trimester of pregnancy, } \\
\text { W/O metabolic disease }\end{array}$ & 185 & NA \\
\hline 9 & Chung et al. [47], 2016 & 3A; DBSI & 12 weeks & BMI 25-35 & 37 & Adult \\
\hline 10 & Zarrati et al. [52], 2013 & 3A; DBSI & 8 weeks & 24 men and 51 women, age $20-50$, BMI 25-35 & 75 & Adult \\
\hline 11 & Lindsay et al. [43], 2014 & 2A; DBSI & 4 weeks & $\begin{array}{l}\text { Pregnant women, }<20 \text { weeks of gestation, } \\
\text { BMI } 30.0-39.9, \text { singleton pregnancy }\end{array}$ & 138 & Adult \\
\hline 12 & Gøbel et al. [50], 2011 & 2A; DBSI & 12 weeks & $\begin{array}{l}\text { BMI percentile which pass through } \\
18.5-25 \mathrm{~kg} / \mathrm{m}^{2} \text { and above } 30 \mathrm{~kg} / \mathrm{m}^{2}\end{array}$ & 50 & $12-15$ \\
\hline 13 & Kadooka et al. [53], 2010 & 2A; DBSI & 12 weeks & $\begin{array}{l}\text { BMI 24.2-30.7, and abdominal } \\
\text { visceral fat area } 81.2-178.5 \mathrm{~cm}^{2}\end{array}$ & 87 & $33-63$ \\
\hline 14 & Hariri et al. [54], 2015 & 2A; DBSI & 8 weeks & $\begin{array}{l}\text { Fasting blood glucose } \geq 126 \mathrm{mg} / \mathrm{dL}, \\
\text { and blood sugar }(2 \mathrm{~h} \text { postprandial }) \geq 200\end{array}$ & 40 & $25-65$ \\
\hline 15 & Ivey et al. [64], 2015 & $4 \mathrm{~A} ; \mathrm{DBSI}$ & 6 weeks & $\begin{array}{l}\mathrm{BMI}>25 \text {, and elevated waist circumference, } \\
\text { and } \mathrm{BP}>120 / 80 \mathrm{~mm} \mathrm{Hg}\end{array}$ & 156 & $>55$ \\
\hline 16 & Lee et al. [55], 2014 & 2A; DBSI & 8 weeks & $\begin{array}{l}\text { Female, BMI }>25 \text {, and waist } \\
\text { circumference }>85 \mathrm{~cm}\end{array}$ & 36 & $19-65$ \\
\hline 17 & Ivey et al. [66], 2014) & 4A; DBSI & 6 weeks & $\begin{array}{l}\mathrm{BMI}>25 \text {, and elevated waist } \\
\text { circumference, and } \mathrm{BP}>120 / 80 \mathrm{~mm} \mathrm{Hg}\end{array}$ & 156 & $>55$ \\
\hline 18 & Gomes et al. [56], 2017 & 2A DBSI & 8 weeks & BMI 24.9-40 & 43 & $20-59$ \\
\hline
\end{tabular}

DBSI, double-blinded (subject and investigator); SBS, single-blinded (subject); 2A, 3A, 4A, and 5A, 2, 3, 4, 5 treatment arms.

BMI, body mass index; BP, blood pressure; NA, not applicable.

comparison, the subjects in the control group typically took foods without probiotics or took placebos. The placebos were similar to the probiotic foods, pills, or capsules in look and taste. The probiotics that were used in the intervention groups were from the Lactobacillus and
Bifidobacterium genera, which include L. gasseri, L. plantarum, L. acidophilus, B. bifidum, L. paracasei, $L$. rhamnosus, $L$. reuteri, $L$. case $i, L$. salivarius, and B. animalis subsp lactis (Table 2). 
Table 2. Description of treatment group(s) and control group(s)

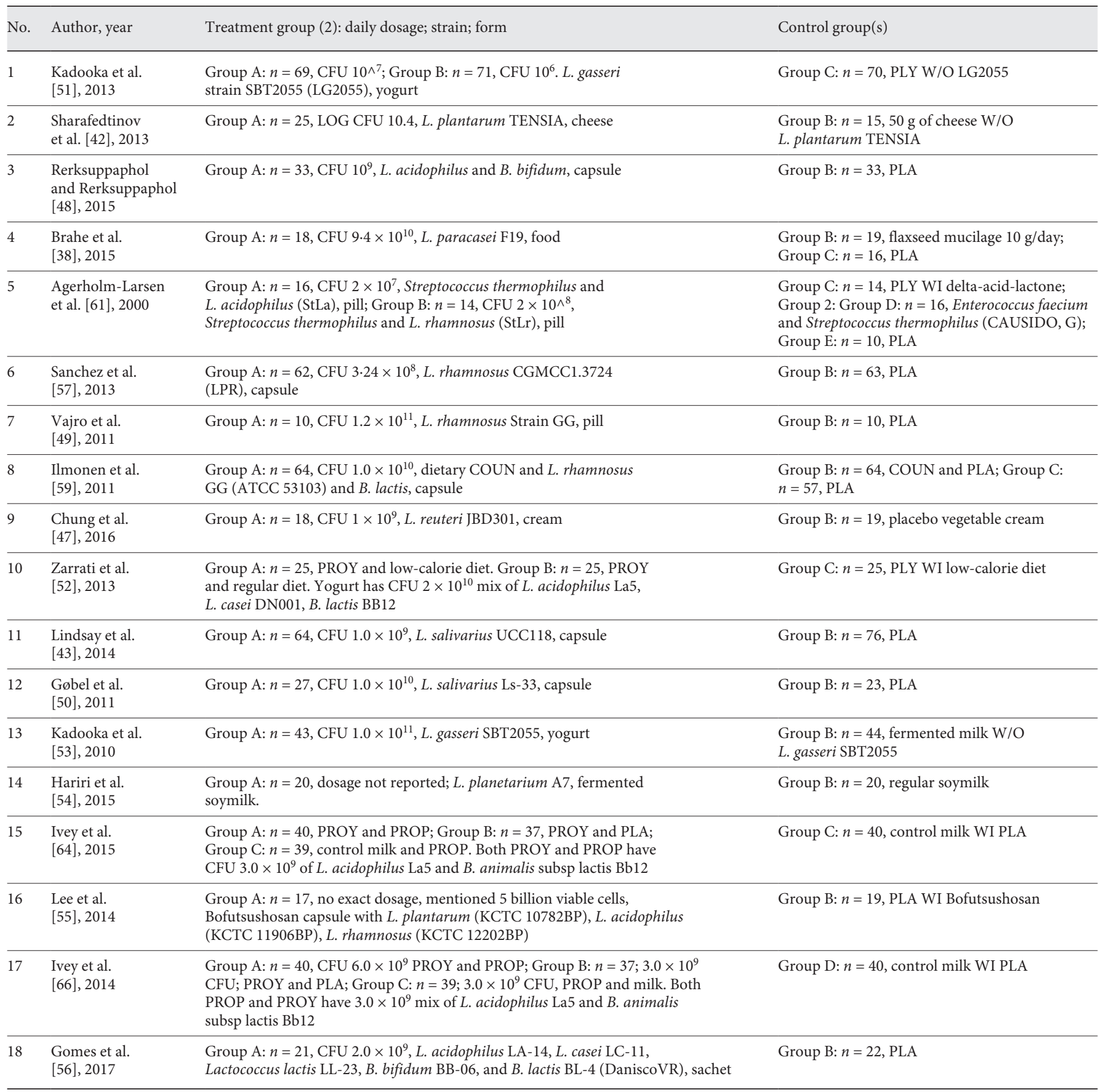

L, Lactobacillus, B, Bifidobacterium; WI, with, W/O, without; PLA, placebo capsules or pills; PLY, placebo yogurt; PROP, probiotic capsules or pills; PROY, probiotic yogurt; COUN, dietary counselling.

\section{Change of Anthropometric Indicators}

In a recent study, Sagun et al. [44] suggested that visceral fat, waist circumferences, hip circumferences, and waist-to-hip ratio were useful nonintrusive and nonbiochemical (e.g., plasma glucose, lipids, uric acid, insulin, and $\mathrm{HbA1c}$ ) measurements when determining whether individuals were at risk of developing MetS. Also, Mooney et al. [45] suggested that in clinical settings, when combining BMI with other anthropometric measurements of body compositions, physicians and health professionals 
Table 3. Result of anthropometric changes and biochemical changes

\begin{tabular}{|c|c|c|c|c|c|}
\hline No. & Author, year & $\begin{array}{l}\text { Significant anthropometric } \\
\text { changes }\end{array}$ & $\begin{array}{l}\text { Nonsignificant } \\
\text { anthropometric changes }\end{array}$ & $\begin{array}{l}\text { Significant biochemical } \\
\text { changes }\end{array}$ & $\begin{array}{l}\text { Nonsignificant } \\
\text { biochemical changes }\end{array}$ \\
\hline 1 & $\begin{array}{l}\text { Kadooka } \\
\text { et al. [51], } 2013\end{array}$ & $\begin{array}{l}\text { Group A vs. C: BMI; waist and hip } \\
\text { CIR, BFM, and BFP } \\
\text { Group B vs. C: BMI; waist and } \\
\text { hip CIR, BFM, and BFP } \\
\text { Within both Group A and B: BMI; } \\
\text { waist and hip CIR, BFM, and BFP }\end{array}$ & NR & NR & NR \\
\hline 2 & $\begin{array}{l}\text { Sharafedtinov } \\
\text { et al. [42], } 2013\end{array}$ & $\begin{array}{l}\text { Group A vs. B: weight } \\
\text { and BMI }\end{array}$ & $\begin{array}{l}\text { Group A vs. B: Fat and } \\
\text { muscle mass or WHR }\end{array}$ & NR & $\begin{array}{l}\text { Group A vs. B: plasma glucose, } \\
\text { plasma lipids, or cholesterol levels }\end{array}$ \\
\hline 3 & $\begin{array}{l}\text { Rerksuppaphol and } \\
\text { Rerksuppaphol [48], } 2015\end{array}$ & NR & $\begin{array}{l}\text { Group A vs. B: Weight, } \\
\text { BMI, or waist and hip CIR }\end{array}$ & $\begin{array}{l}\text { Group A vs. B: TC, } \\
\text { LDL-C, and HDL-C }\end{array}$ & $\begin{array}{l}\text { Group A vs B: serum TAG or } \\
\text { fasting blood glucose levels }\end{array}$ \\
\hline 4 & Brahe et al. [38], 2015 & NR & NR & $\begin{array}{l}\text { Group A vs. B, A vs. } \\
\text { C, B vs. C: insulin AUC, } \\
\text { Group A vs. B: LDL-C } \\
\text { Group A vs. B.: LDL-C }\end{array}$ & $\begin{array}{l}\text { Fasting blood glucose, TC } \\
\text { HDL-C or fasting insulin } \\
\text { level in any comparison }\end{array}$ \\
\hline 5 & $\begin{array}{l}\text { Agerholm-Larsen } \\
\text { et al. [61], } 2000\end{array}$ & $\begin{array}{l}\text { Group A vs. B: systolic BP, } \\
\text { Group D vs. B: systolic BP, } \\
\text { Within Group A, D, and E: weight, } \\
\text { Within Group A, D, and E: BFM, } \\
\text { Within Group A and D: systolic BP, } \\
\text { Within Group A and E; diastolic BP }\end{array}$ & NR & $\begin{array}{l}\text { Group D vs. C, and } \\
\text { Group D vs. E: LDL-C }\end{array}$ & $\begin{array}{l}\text { TC, HDL-C, or triacylglycerol } \\
\text { in any comparison }\end{array}$ \\
\hline 6 & $\begin{array}{l}\text { Sanchez } \\
\text { et al. [57], } 2013\end{array}$ & $\begin{array}{l}\text { Group A vs. B: weight and } \\
\text { BFM among women }\end{array}$ & $\begin{array}{l}\text { Group A vs. B: weight and } \\
\text { BFM for total population }\end{array}$ & NR & $\begin{array}{l}\text { Group A vs. B: TC, HDL-C, } \\
\text { fasting glucose or fasting } \\
\text { insulin level }\end{array}$ \\
\hline 7 & Vajro et al. [49], 2011 & NR & $\begin{array}{l}\text { Group A vs. B: BMI or } \\
\text { visceral fat area }\end{array}$ & NR & NR \\
\hline 8 & Ilmonen et al. [59], 2011 & $\begin{array}{l}\text { Group A vs. B, and } \\
\text { Group A vs. C: waist CIR }\end{array}$ & NR & $\begin{array}{l}\text { Group A vs. B, Group A vs. C, } \\
\text { Group B vs. C: postpartum } \\
\text { glucose, mmol/L }\end{array}$ & NR \\
\hline 9 & Chung et al. [47], 2016 & $\begin{array}{l}\text { Group A vs. B: percentage } \\
\text { change in weight and BMI }\end{array}$ & NR & NR & $\begin{array}{l}\text { Group A vs. B: blood lipid profile, } \\
\text { fasting blood glucose, blood } \\
\text { insulin, or HbAlc }\end{array}$ \\
\hline 10 & Zarrati et al. [52], 2013 & $\begin{array}{l}\text { Group A vs. C, and Group B vs. C: } \\
\text { weight, BMI, waist CIR, and hip CIR }\end{array}$ & $\begin{array}{l}\text { WHR, SBP, or DBP in } \\
\text { any comparison }\end{array}$ & NR & NR \\
\hline 11 & Lindsay et al. [43], 2014 & NR & NR & NR & $\begin{array}{l}\text { Group A vs. B: fasting glucose } \\
\text { level, insulin, TC HDL-C, } \\
\text { LDL-C, or TAG }\end{array}$ \\
\hline 12 & Gøbel et al. [50], 2011 & NR & $\begin{array}{l}\text { Group A vs. B: BMI, } \\
\text { WHR, BFP, BP }\end{array}$ & NR & $\begin{array}{l}\text { Group A vs. B: fasting glucose, } \\
\text { insulin, TC, HDL-C, LDL-C, } \\
\text { TAG }\end{array}$ \\
\hline 13 & $\begin{array}{l}\text { Kadooka } \\
\text { et al. [53], } 2010\end{array}$ & $\begin{array}{l}\text { Group A vs. B: BMI, weight, waist } \\
\text { and hip CIR, WHR } \\
\text { Within Group A: BMI, } \\
\text { weight, waist and hip CIR }\end{array}$ & NR & NR & $\begin{array}{l}\text { Group A vs. B: TAG, TC, } \\
\text { HDL-C, or LDL-C }\end{array}$ \\
\hline 14 & $\begin{array}{l}\text { Hariri } \\
\text { et al. [54], } 2015\end{array}$ & $\begin{array}{l}\text { Group A vs. B: systolic BP } \\
\text { and diastolic BP, } \\
\text { Within Group A: weight, BMI, } \\
\text { WHR, systolic BP and diastolic BP, } \\
\text { Within Group B: weight and BMI }\end{array}$ & $\begin{array}{l}\text { Group A vs. B: weight, } \\
\text { BMI, or WHR }\end{array}$ & NR & NR \\
\hline 15 & Ivey et al. [64], 2015 & NR & $\begin{array}{l}\text { Group A vs. C, } \\
\text { Group B vs. C: BP }\end{array}$ & NR & $\begin{array}{l}\text { Group A vs. C, Group B vs. C: } \\
\text { TC, HDL-C, LDL-C or TAG }\end{array}$ \\
\hline 16 & Lee et al. [55], 2014 & $\begin{array}{l}\text { Within Group A and B: weight, } \\
\text { BMI, waist CIR, BFP, BFM }\end{array}$ & $\begin{array}{l}\text { Group A vs. B: weight, } \\
\text { BMI, waist CIR, BFP, } \\
\text { or BFM }\end{array}$ & $\begin{array}{l}\text { Group A vs. B: HDL-C, } \\
\text { Within Group B: blood } \\
\text { glucose level, } \\
\text { HDL-C, and TC }\end{array}$ & $\begin{array}{l}\text { Group A vs. B: blood glucose } \\
\text { level, TAG, or TC }\end{array}$ \\
\hline 17 & Ivey et al. [66], 2014 & NR & NR & Group B vs D: blood glucose & $\begin{array}{l}\text { Group A vs. C: HbA1c, blood } \\
\text { glucose, or insulin, } \\
\text { Group B vs. D: HbA1c or insulin }\end{array}$ \\
\hline
\end{tabular}


Table 3. (continued)

\begin{tabular}{lllll}
\hline No. Author, year & $\begin{array}{l}\text { Significant anthropometric } \\
\text { changes }\end{array}$ & $\begin{array}{l}\text { Nonsignificant } \\
\text { anthropometric changes }\end{array}$ & $\begin{array}{l}\text { Significant biochemical } \\
\text { changes }\end{array}$ \\
\hline $18 \quad$ Gomes et al. [56], 2017 & $\begin{array}{l}\text { Group A vs. B: waist CIR, } \\
\text { waist-height ratio, conicity index }\end{array}$ & NR & $\begin{array}{l}\text { Group A vs. B: decrease in } \\
\text { plasma polyunsaturated } \\
\text { fatty acids, increase in the } \\
\text { activity of glutathione } \\
\text { peroxidase }\end{array}$ \\
\hline
\end{tabular}

Cir, circumferences; BFM, body fat mass; BFP, body fat percentage; BP, blood pressure; WHR, waist-to-hip ratio; TAG, triglyceride; NR, not reported; LDL-C, low-density lipoprotein cholesterol; HDL-C, high-density lipoprotein cholesterol; TC, total cholesterol.

\begin{tabular}{|c|c|c|c|c|c|c|c|c|c|c|c|}
\hline \multirow{3}{*}{$\frac{\text { Study or subgroup }}{\text { Gomes et al., } 2017}$} & \multicolumn{2}{|c|}{ Experimental } & \multicolumn{4}{|c|}{ Control } & \multicolumn{2}{|r|}{ Std. mean difference } & \multirow{2}{*}{\multicolumn{2}{|c|}{$\begin{array}{l}\text { Std. mean difference } \\
\mathrm{IV}, \text { random, } 95 \% \mathrm{Cl}\end{array}$}} & \\
\hline & \multirow{2}{*}{$\begin{array}{l}\text { Mean } \\
-0.45\end{array}$} & \multirow{2}{*}{$\begin{array}{r}\text { SD } \\
3.93\end{array}$} & \multirow{2}{*}{$\begin{array}{r}\text { Total } \\
21\end{array}$} & \multirow{2}{*}{$\frac{\text { Mean }}{-0.72}$} & \multirow{2}{*}{$\begin{array}{r}\text { SD } \\
4.61\end{array}$} & \multirow{2}{*}{$\begin{array}{r}\text { Total } \\
22\end{array}$} & \multirow{2}{*}{$\begin{array}{r}\text { Weight } \\
11.6 \%\end{array}$} & \multirow{2}{*}{$\begin{array}{r}\text { IV, random, } 95 \% \mathrm{Cl} \\
0.06[-0.54,0.66]\end{array}$} & & & \\
\hline & & & & & & & & & & & \\
\hline Hariri et al., 2015 & -0.03 & 9.67 & 20 & -0.25 & 10.81 & 20 & $11.3 \%$ & $0.02[-0.60,0.64]$ & & & \\
\hline Kadooka et al., 2010 & -0.4 & 0.5 & 43 & 0.1 & 0.51 & 44 & $13.6 \%$ & $-0.98[-1.43,-0.54]$ & 1 & & \\
\hline Kadooka et al., 2013 & -0.3507 & 0.4265 & 140 & 0.1 & 0.64 & 70 & $15.6 \%$ & $-0.89[-1.18,-0.59]$ & $\leftarrow$ & & \\
\hline Lee et al., 2014 & 0.38 & 0.67 & 17 & 0.75 & 0.52 & 19 & $10.6 \%$ & $-0.61[-1.28,0.06]$ & & - & \\
\hline Sanchez et al., 2017 & -1.88 & 10.25 & 45 & -1.38 & 10.31 & 48 & $14.2 \%$ & $-0.05[-0.45,0.36]$ & & & \\
\hline Sharafedtinov et al., 2013 & -2 & 4.07 & 25 & -1.6 & 4.25 & 15 & $11.0 \%$ & $-0.09[-0.74,0.55]$ & & & \\
\hline Zarrati et al., 2013 & -1.55 & 6.33 & 25 & -1.9 & 6.63 & 25 & $12.1 \%$ & $0.05[-0.50,0.61]$ & & & \\
\hline Total $(95 \% \mathrm{Cl})$ & & & 336 & & & 263 & $100.0 \%$ & $-0.34[-0.68,0.01]$ & & & \\
\hline Heterogeneity: $\mathrm{Tau}^{2}=0.18$ & 8; $\mathrm{Chi}^{2}=2$ & 26.89, df & $f=7(p$ & $D=0.00$ & $003) ; 1^{2}$ & $=74 \%$ & & & & & \\
\hline Test for overall effect: $\mathrm{Z}$ = & $1.91(p=$ & $0.06)$ & & & & & & & -0.5 & 0.5 & 1 \\
\hline & & & & & & & & & Favours (experimental) & Favours (co & \\
\hline
\end{tabular}

Fig. 2. Comparison of standardized mean net changes of BMI between probiotic groups and control groups. $\mathrm{Tau}^{2}=0.18, I^{2}=74 \%, 95 \% \mathrm{CI}-0.68$ to $0.01, Z=1.91, p=0.06$. No significant difference in the comparison of standardized mean net changes of BMI between probiotic groups and control groups.

could get detailed information about individuals' MetSrelated health risks. Moreover, when diagnosing MetS, the costs of anthropometric measurements were lower than using biochemical measurements [44]. Thus, for health professionals who work in low-income communities or under financial constraints, they could use anthropometric measurements to meet the needs of large epidemiological studies, routine medical practice, or both.

$B M I$ was calculated as the ratio of weight in kilograms divided by the square of the height in meters. A BMI of $25 \mathrm{~kg} / \mathrm{m}^{2}$ or higher was considered overweight, and a BMI of $30 \mathrm{~kg} / \mathrm{m}^{2}$ or higher was considered obese [46]. BMI was mentioned as an outcome measurement in 12 studies, which were comprised with 792 study participants. After further examination, 4 studies were excluded from the meta-analysis (Chung et al. [47], no original data; Rerksuppaphol and Rerksuppaphol [48], data of BMI was not provided, used BMI z-score, not BMI [49]; Gøbel et al. [50], used BMI-for-age z-score, not BMI).

Probiotics and MetS, A Meta-Analysis
The remaining 8 studies were further analyzed. Four studies [42, 51-53] reported significant differences in BMI between intervention and control group. While the rest of the studies [54-57] did not find significant BMI changes after the intervention.

When combining the findings from these 8 studies (599 participants), the result suggested that there is no significant ( $p=0.06$; Fig. 2 ) difference in the comparison of standardized mean net changes of BMI between the control group(s) and the probiotic intervention group(s) groups who used Lactobacillus (L. planetarium, L. gasseri, L. plantarum, L. acidophilus, L. rhamnosus, L. rhamnosus, L. acidophilus, L. casei) and Bifidobacterium (B. lactis) foods or supplements.

Waist, hip circumference, and waist-to-hip ratio are 3 other useful MetS measurements. Janssen et al. [58] suggested that because waist, hip circumference, and waistto-hip ratio likely reflect the abdominal, and in particular, visceral fat; thus, using these 3 anthropometric measure-

Ann Nutr Metab 2019;74:224-241 


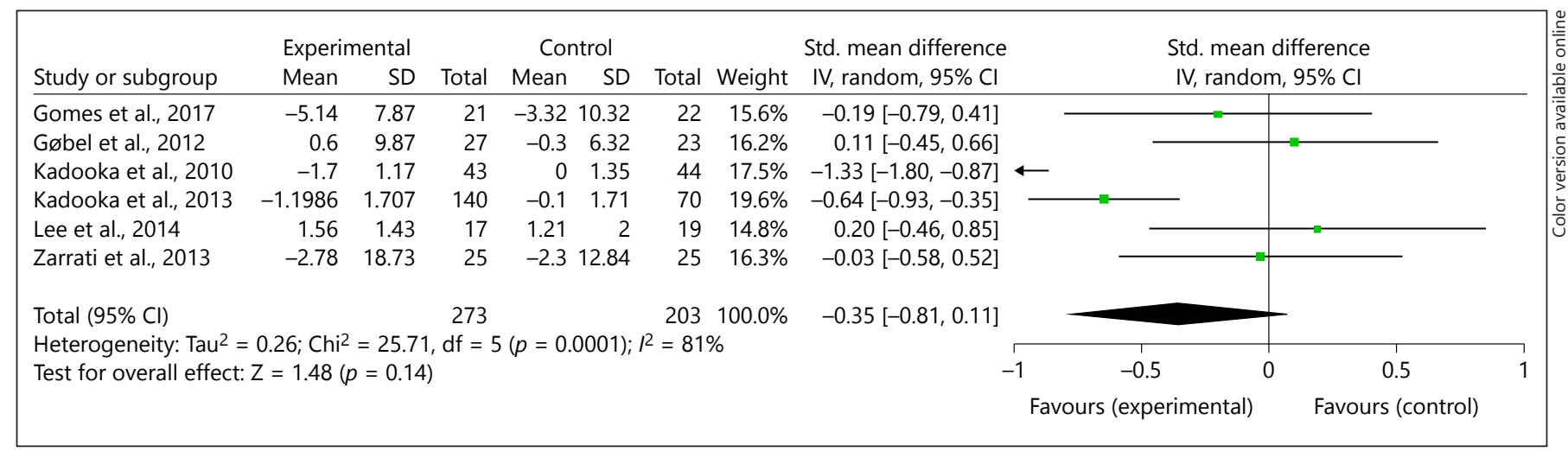

Fig. 3. Comparison of standardized mean net changes of waist circumference between probiotic groups and control groups. Tau $^{2}=0.26, I^{2}=81 \%, 95 \%$ CI -0.81 to $0.11, Z=1.48, p=0.14$. No significant difference in the comparison of standardized mean net changes of waist circumference.

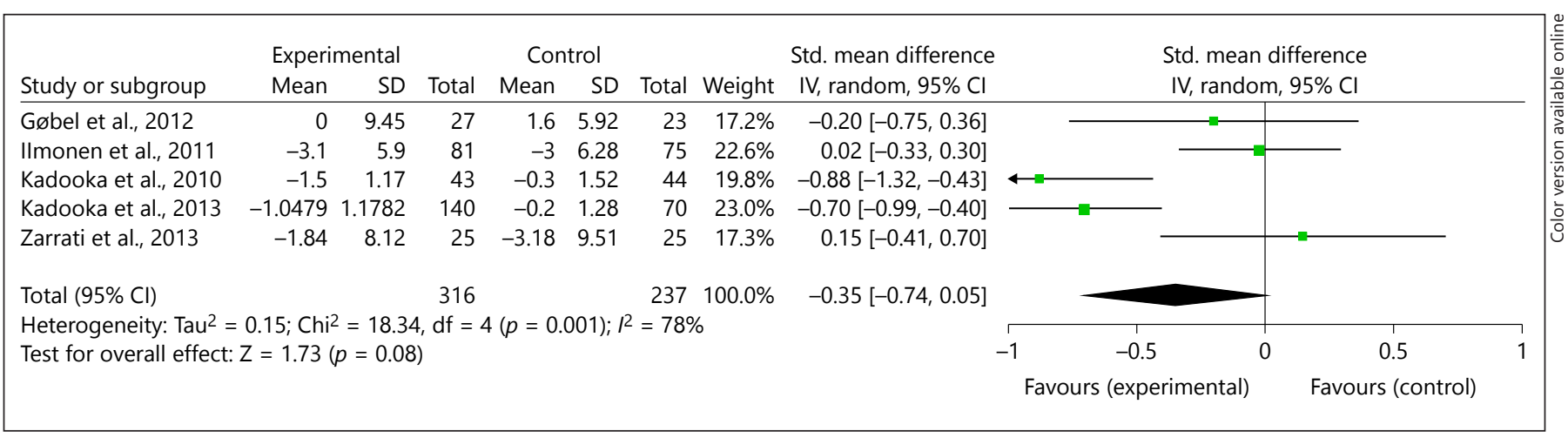

Fig. 4. Comparison of standardized mean net changes of hip circumference between probiotic groups and control groups. Tau $^{2}=0.15, I^{2}=78 \%, 95 \% \mathrm{CI}-0.74$ to $0.05, Z=1.73, p=0.08$. No significant difference in the comparison of standardized mean net changes of hip circumference.

ments could better explain the health risk of MetS better than using BMI alone. Six reviewed studies showed significant differences in the waist circumference, hip circumference, or both $[51-53,55,56,59]$. While other 5 studies found no significant changes in these anthropometric outcome measurements $[42,48,50,52,54]$.

As shown in Figure 3, in the 6 reviewed studies (476 participants), compared to the control group, the participants who received Lactobacillus and Bifidobacterium foods or supplements did not experienced a significant changes in their waist circumferences $(p=0.14)$. Similarly, in the 5 reviewed studies (553 participants), probiotics intervention did not cause significant change in hip circumference ( $p=0.08$; Fig. 4$)$. In addition, as shown in Figure 5 , in the 5 studies that measured waist-to-hip ratio (267 participants), there was no significant change between the intervention groups and the control groups $(p=0.62)$.
$B F M$ and $B F P$ are anthropometric indices that are directly related to the risk of developing MetS [60]. Among reviewed studies, 2 studies suggested that there were significant differences in reduction of BFM, BFP, or both $[51,61]$, and the other 5 studies did not find significant differences in changes of BFM or BFP [42, 50, 55-57]. In the 7 reviewed studies (569 participants), the results suggested that there was no significant difference between the intervention groups and the control groups for BFM ( $p=0.07$, Fig. 6).

However, in the 6 reviewed studies (519 participants), the result suggested that there is a significant difference in the standardized mean net changes of fat percentage (BFP) between probiotic groups and control groups (95\% CI -0.58 to $-0.02 ; p=0.04 ;$ Fig. 7 ). The standardized mean difference is -0.30 , which suggested that the mean of the intervention group is $-0.30 \mathrm{SD}$ away from the mean of the 


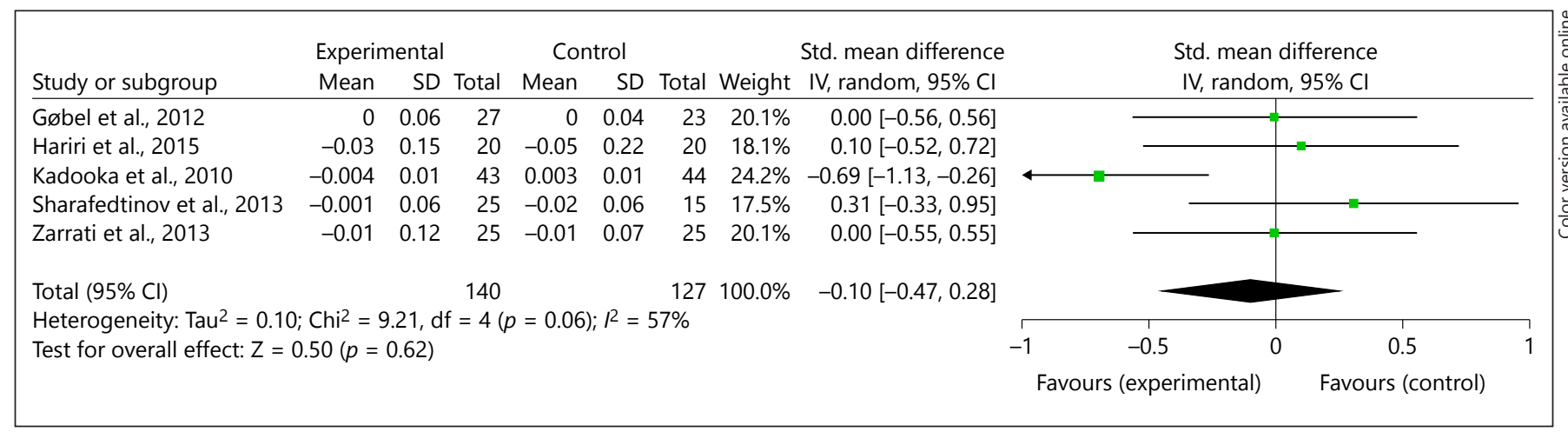

Fig. 5. Comparison of standardized mean net changes of waist-to-hip ratio between probiotic groups and control groups. $\mathrm{Tau}^{2}=0.10, I^{2}=57 \%, 95 \% \mathrm{CI}-0.47$ to $0.28, Z=0.50, p=0.62$. No significant difference in the comparison of standardized mean net changes of waist-to-hip ratio.

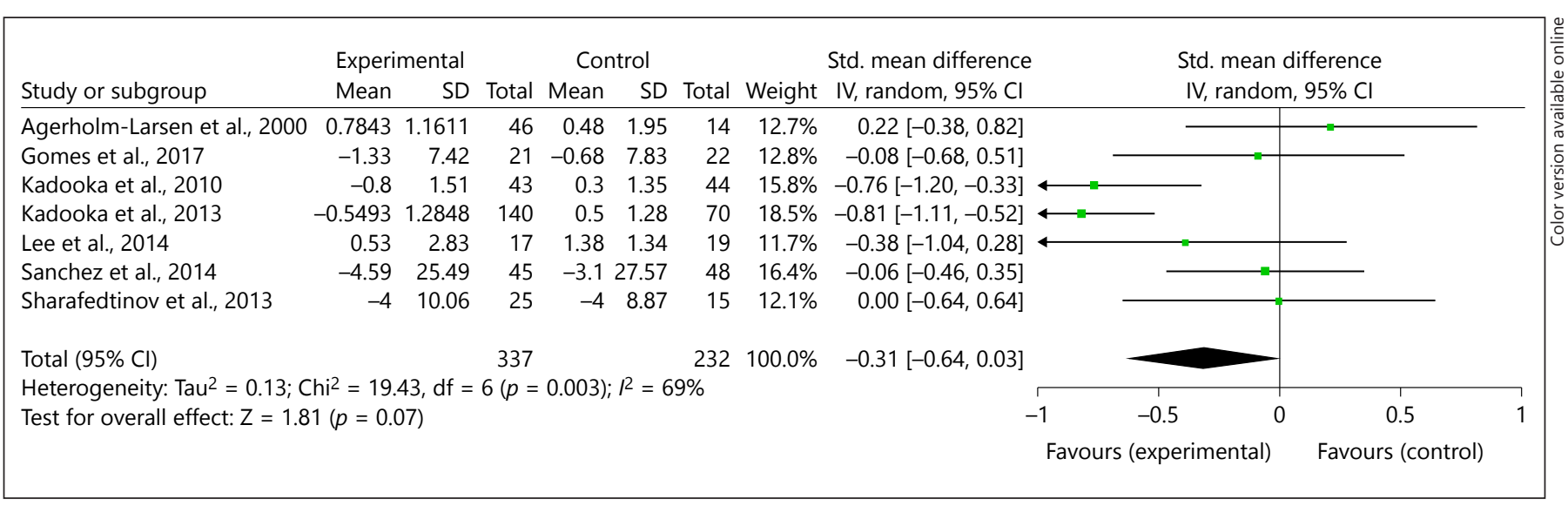

Fig. 6. Comparison of standardized mean net changes of BFM between probiotic groups and control groups. $\mathrm{Tau}^{2}=0.13, I^{2}=69 \%, 95 \% \mathrm{CI}-0.64$ to $0.03, Z=1.81, p=0.07$. No significant difference in the comparison of standardized mean net changes of BFM.

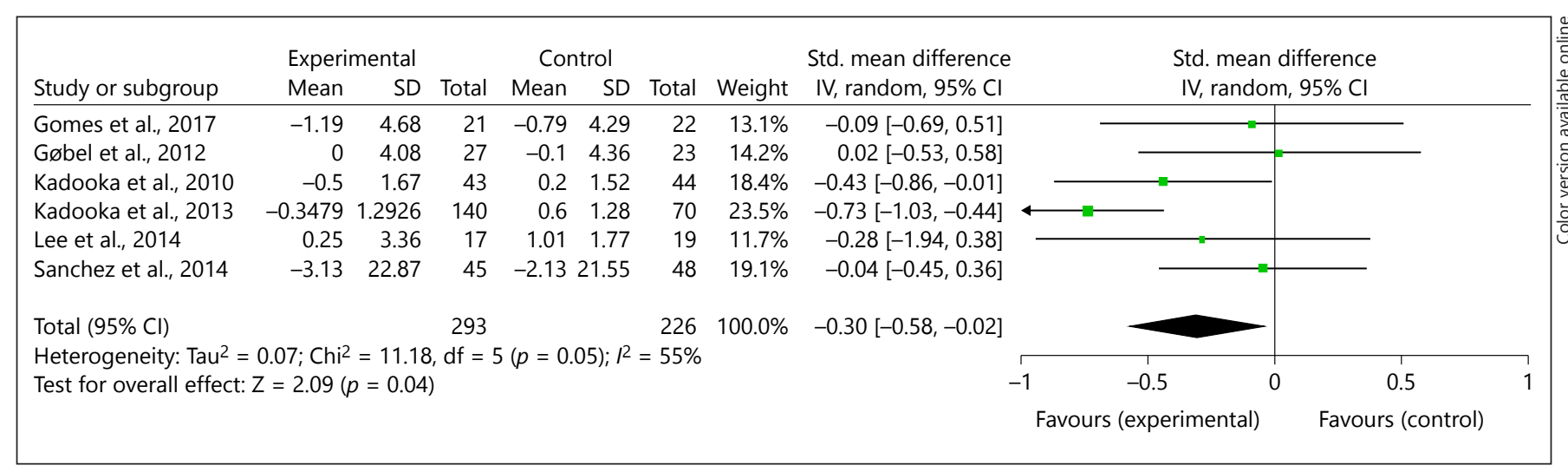

Fig. 7. Comparison of standardized mean net changes of BFP between probiotic groups and control groups. $\mathrm{Tau}^{2}=0.07, I^{2}=55 \%$, $95 \%$ CI -0.58 to $-0.02, Z=2.09, p=0.04<0.05$. Significant differ- ence in the comparison of standardized mean net changes of BFP. Standardized mean difference $=-0.30$, which indicates intervention has a small to medium in effect reducing BFP. 


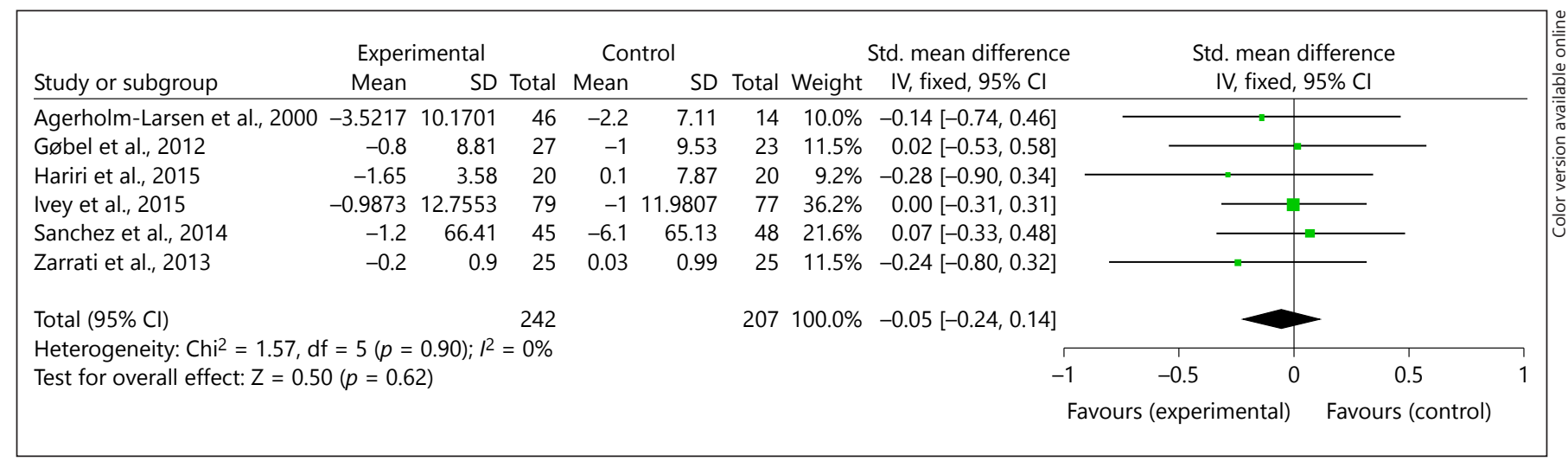

Fig. 8. Comparison of standardized mean net changes of SBP between probiotic groups and control groups. $I^{2}=$ $0 \%, 95 \%$ CI -0.24 to $0.14, Z=0.50, p=0.62>0.05$. No significant difference in the comparison of standardized mean net changes of SBP.

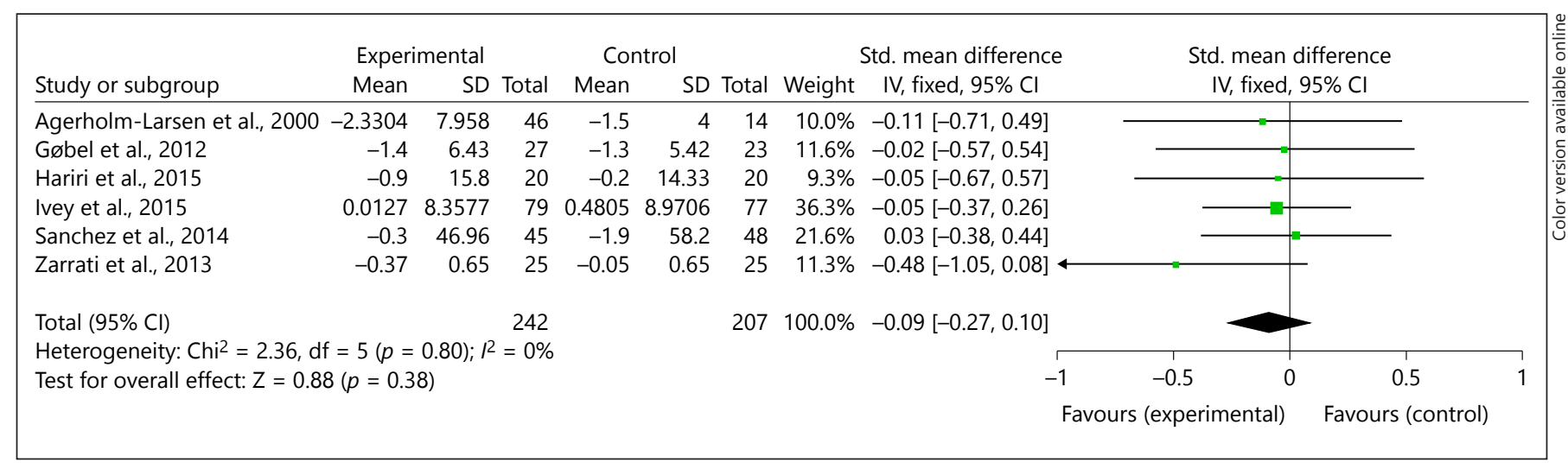

Fig. 9. Comparison of standardized mean net changes of DBP between probiotic groups and control groups. $I^{2}=$ $0 \%, 95 \%$ CI -0.27 to $0.10, Z=0.88, p=0.38>0.05$. No significant difference in the comparison of standardized mean net changes of DBP.

control group. In terms of clinical meaning, as it was suggested by Cohen [39], this result suggested the intervention had a small to medium effect on the outcome.

$D B P$ and $S B P$ are usually higher among individuals with MetS than those without MetS [62]. Untreated diastolic or systolic hypertension significantly increases the risk of cardiovascular death [63]. In the reviewed studies, only Hariri et al. [54] reported a significant decrease of DBP and SBP when participants took Lactobacillus and Bifidobacterium foods or supplements. Other studies $[50,52,57,64]$ reported no significant differences in blood pressure. Also, Agerholm-Larsen et al. [61] suggested that there was a significant decrease in SBP and a significant increase in DBP between the intervention group and the control group. As it was shown in Figure
8, when combined the results from 6 studies (449 participants), there was no significant differences in SBP $(p=0.62)$. Similarly, there was no significant difference in DBP ( $p=0.38$; Fig. 9) between the intervention and the control groups.

\section{Change of Biochemical Indicators}

Abnormal blood values were determined in accordance with the criteria for the MetS as defined by the National Cholesterol Education Program [65]. Patients who have MetS tend to have unusual value in metabolic-related clinical biochemical markers, such as abnormal fasting glucose, fasting insulin, lipid profile, and $\mathrm{HbA1c}$.

Fasting glucose is a commonly used indicator to determine whether a patient has diabetes or is prediabetic. Ten 


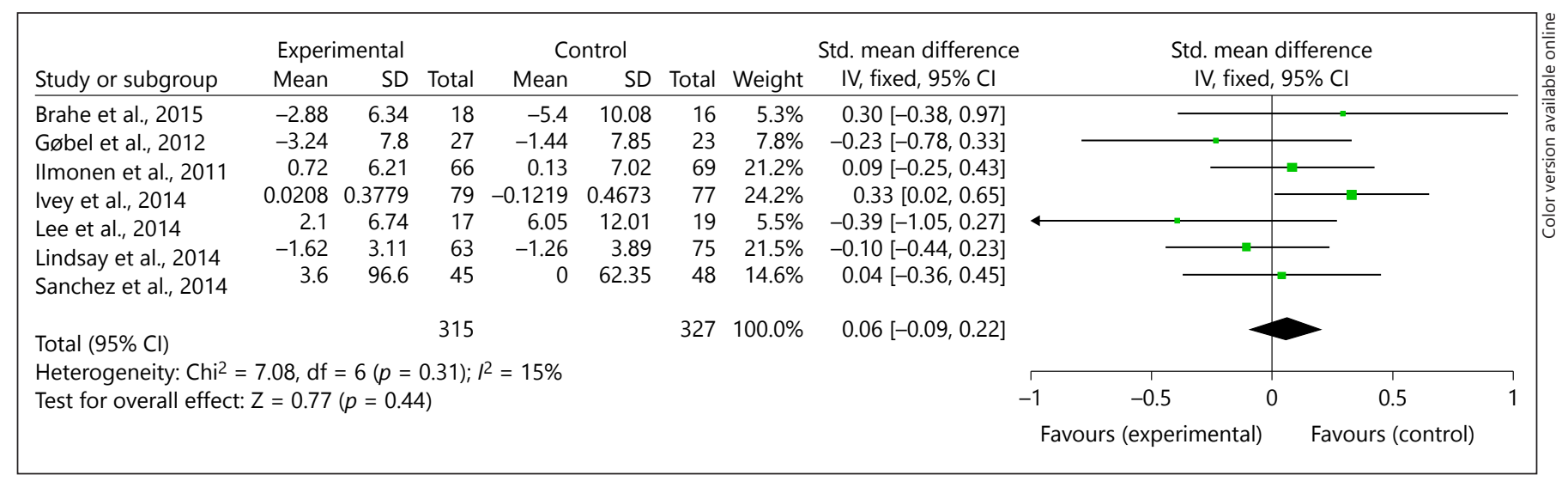

Fig. 10. Comparison of standardized mean net changes of fasting glucose between probiotic groups and control groups. $I^{2}=15 \%, 95 \% \mathrm{CI}=-0.09$ to $0.22, Z=0.77, p=0.44>0.05$. No significant difference in the comparison of standardized mean net changes of fasting glucose level.

studies discussed the efficacy of Lactobacillus food and/or supplement intake on fasting glucose. Upon further examination, 3 studies were removed from the meta-analysis. The reasons for the removal include lack of original data [47], data without specific SD of fasting glucose [48], and outcome indicator being serum glucose, not fasting glucose [42]. Among the remaining 7 studies, 5 of them found no significant differences in fasting glucose or blood glucose in between-group comparisons [ $38,43,50,55,57]$. In the 2014 4-arm study by Ivey et al. [66], the researchers used a 4-arm approach: (A) probiotic yogurt plus probiotic capsules, (B) probiotic yogurt plus placebo capsules, (C) control milk plus probiotic capsules, and (D) control milk plus placebo capsules. The author found significant differences in blood glucose between group B and D $(p<$ 0.037 ), and no significant difference was found between group $\mathrm{A}$ and $\mathrm{C}$. To conduct the meta-analysis, the reviewers standardized the unit-of-measurement prior to computations. The fasting glucose level in $\mathrm{mmol} / \mathrm{L}$ was converted to $\mathrm{mg} / \mathrm{dL}$, and the conversion factor was $1 \mathrm{mmol} / \mathrm{L}=$ $18.0 \mathrm{mg} / \mathrm{dL}$. In the 7 reviewed studies (642 participants), the result suggested that there was no significant difference in the fasting glucose level between the intervention groups and the control groups ( $p=0.44$; Fig. 10 ).

Fasting insulin is another indicator of health for individuals with MetS. An abnormal fasting insulin level indicates a potential risk of developing diabetes; thus, it is crucial for both patients and health-care professionals to take precautionary measures to prevent diabetes when abnormal fasting insulin levels are presented [67]. In the 6 reviewed studies, the authors did not find significant fasting insulin differences between the intervention groups and control groups [38, 43, 47, 50, 57, 66]. Except for the study without original data conducted by Chung et al. [47]. In the study by Brahe et al. [38], the researchers noticed that there was a significant difference of AUC insulin in between group comparisons; however, they did not find any significant difference in fasting insulin in any between group comparisons. Similarly to the meta-analysis of the fasting glucose level, the reviewers standardized the unitof-measurement prior to computations. The fasting insulin level in $\mathrm{mmol} / \mathrm{L}$ was converted to $\mathrm{mg} / \mathrm{dL}$ prior to computations, and the conversion factor was $1 \mathrm{mmol} / \mathrm{L}=18.0$ $\mathrm{mg} / \mathrm{dL}$. The result of the meta-analysis of the 5 reviewed studies (471 participant) suggested that the probiotic intervention did not have significant effect $(p=0.37$; Fig. 11).

Lipid profile consists of TC, LDL-C, High-HDL-C, and Triglycerides. Regarding the overall effects of probiotic food and supplements on cholesterol levels, only one study showed that interventions had significantly lowered the TC, LDL-C, and HDL-C levels [48]. However, in the other 4 studies, the researchers did not find significant effects on all these 3 (TC, LDL-C, and HDL-C) indicators $[47,54,62,64]$.

Two studies reported significant differences in LDL-C level when they compared the probiotic-intervention group with the control group $[38,61]$. Nevertheless, these 2 studies and the study by Sanchez et al. [57] all failed to find any significant changes in the TC and HDL-C between the intervention groups and control groups. In addition, there were no significant differences observed in the TC level in the other 2 studies $[42,55]$. In the 7 studies that evaluated the changes of the triglycerides level, none of them reported significant differences [42, 43, 48, 50, 55, 56, 64]. 


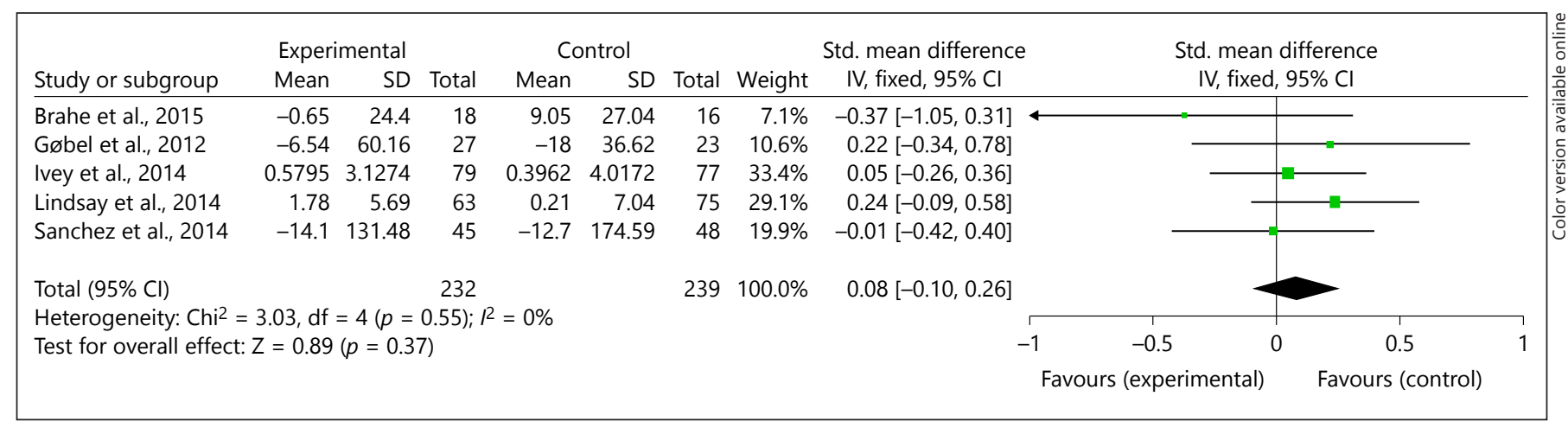

Fig. 11. Comparison of standardized mean net changes of fasting insulin between probiotic groups and control groups. $I^{2}=0 \%, 95 \% \mathrm{CI}=-0.10$ to $0.26, Z=0.89, p=0.37>0.05$. No significant difference in the comparison of standardized mean net changes of fasting insulin level.

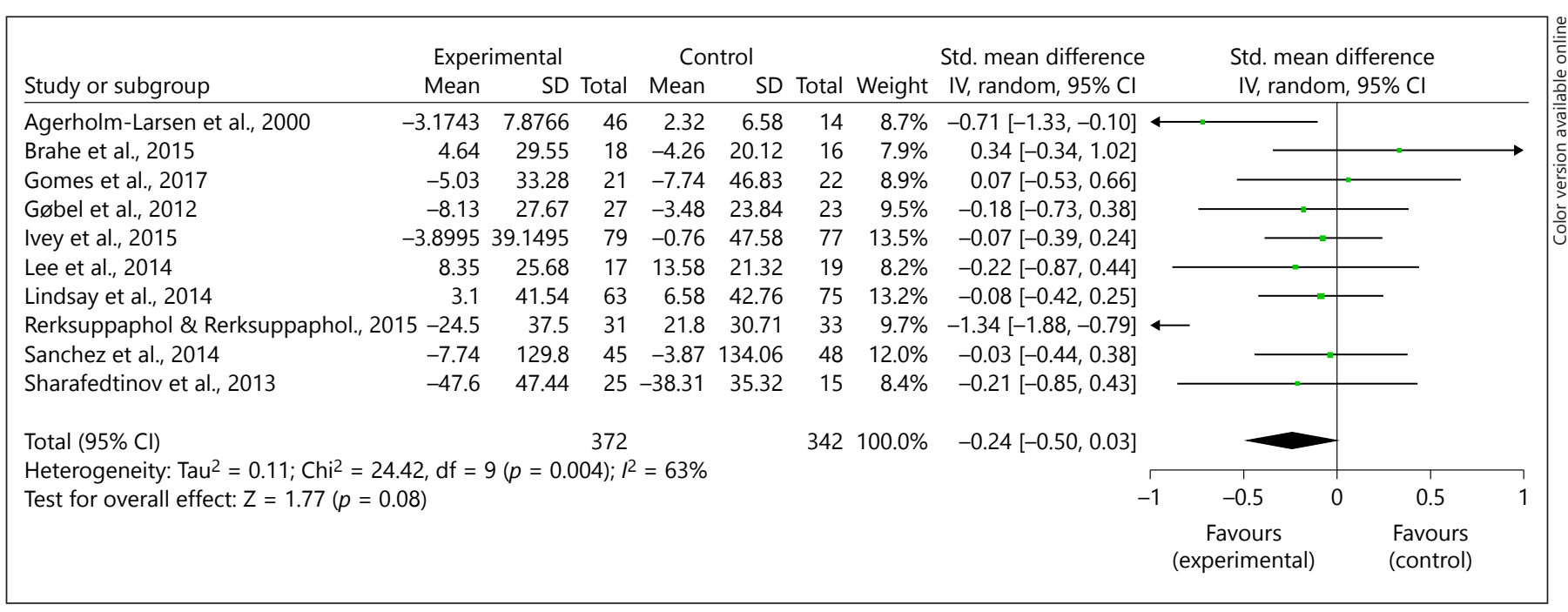

Fig. 12. Comparison of standardized mean net changes of TC between probiotic groups and control groups. $\mathrm{Tau}^{2}=0.11, I^{2}=63 \%, 95 \% \mathrm{CI}-0.50$ to $0.03, Z=1.77, p=0.08>0.05$. No significant difference in the comparison of standardized mean net changes of TC.

In order to perform calculation, the researchers standardized the unit-of-measurement to conduct the metaanalysis, lipid levels in $\mathrm{mmol} / \mathrm{L}$ were converted to $\mathrm{mg} / \mathrm{dL}$, and the conversion factor was $1 \mathrm{mmol} / \mathrm{L}=38.7 \mathrm{mg} / \mathrm{dL}$ for cholesterol and $1 \mathrm{mmol} / \mathrm{L}=88.5 \mathrm{mg} / \mathrm{dL}$ for triglycerides. The mean net changes (mean values $\pm \mathrm{SD}$ ) in the TC, HDL cholesterol, LDL cholesterol, and triglycerides of each study were individually calculated.

As shown in Figure 12, in the 10 reviewed studies (714 participants), there was no significant difference in TC changes between the control group and the intervention group ( $p=0.08$ ). In addition, as it was shown in Figure 13 , there was no significant effect of using probiotics on the HDL-C level $(p=0.29)$.
However, the results in 9 reviewed study (678 participants) showed that probiotic food and supplements had a significant effect on reducing the LDL-C level $(p=0.02$ $<0.05, I^{2}=32,95 \%$ CI -0.34 to $-0.03, Z=2.36$; Fig. 14). It is worth to point out that the effect size is -0.18 , which suggested that the mean of the intervention group is only $-0.18 \mathrm{SD}$ away from the mean of the control group. As it was suggested by Cohen et al. [39], this result suggested the intervention only yield a trivial effect on reducing LDL-C.

In terms of the triglycerides, the meta-analysis of 8 reviewed studies (587 participants) did not find any significant effect between probiotic groups and control groups $(p=0.38$; Fig. 15). 


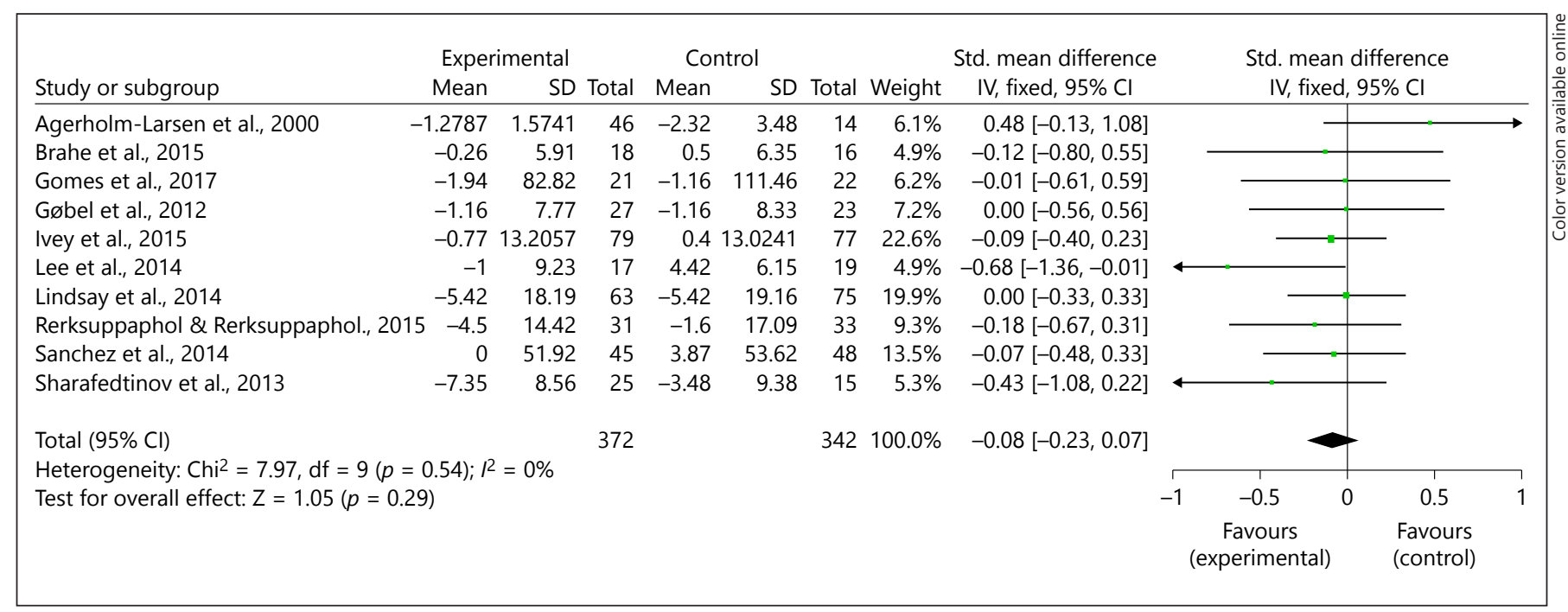

Fig. 13. Comparison of standardized mean net changes of HDL-C between probiotic groups and control groups. $I^{2}=0 \%, 95 \%$ CI -0.23 to $0.07, Z=1.05, p=0.29>0.05$. No significant difference in the comparison of standardized mean net changes of HDL-C.

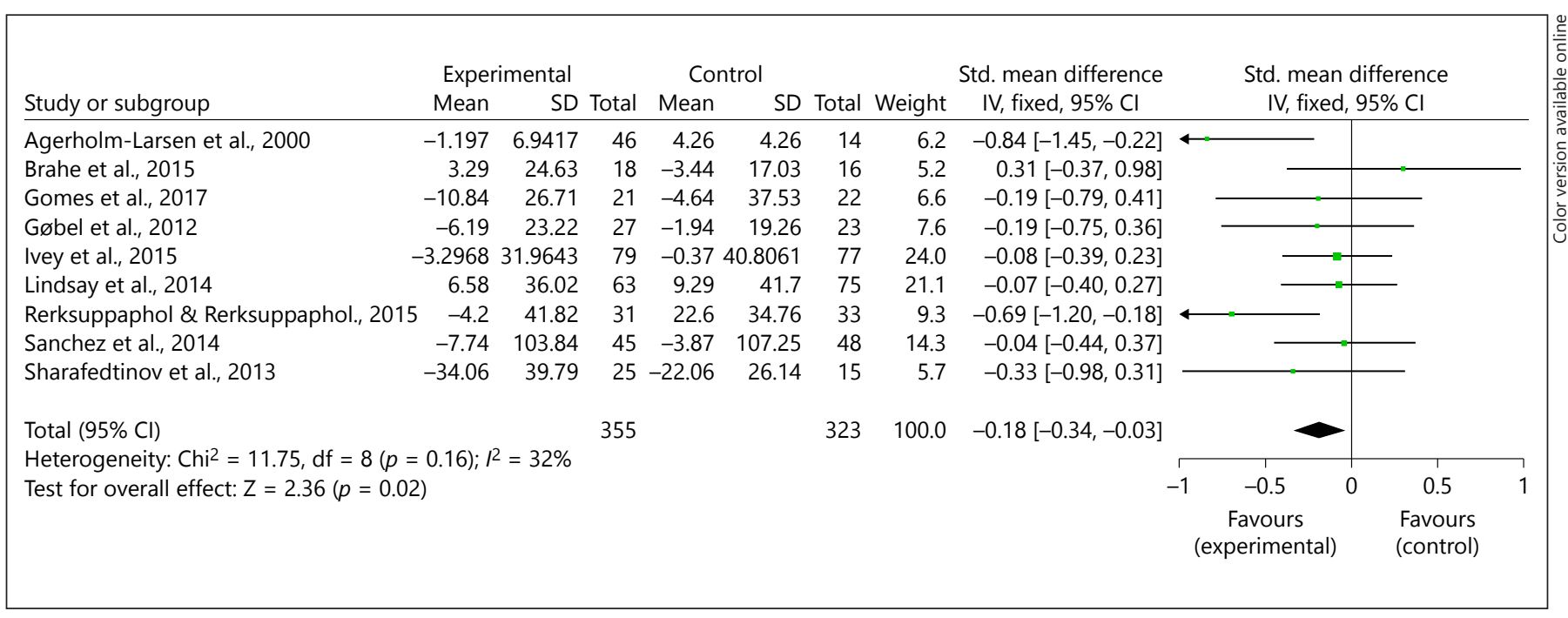

Fig. 14. Comparison of standardized mean net changes of lowdensity lipoprotein cholesterol (LDL-C) between probiotic groups and control groups. $I^{2}=32 \%, 95 \% \mathrm{CI}-0.34$ to $-0.03, Z=2.36, p=$ $0.02<0.05$. Significant difference in the comparison of standard-

$H b A 1 c$ was only evaluated in 3 studies $[47,56,66]$. Since there were no original data in the report of Chung et al. [47]; thus, the meta-analysis was performed based on the remaining 2 studies (199 participants) [56, 66]. The results showed no significant difference in $\mathrm{HbA1c}$ between the probiotic and control groups $(p=0.20$, Fig. 16). ized mean net changes of LDL-C. However, the standardized mean difference $=-0.18$, which indicates intervention only has a trivial effect in reducing LDL-C level.

\section{Discussion}

This review and meta-analysis described and evaluated 18 RCTs studies that used Lactobacillus or Bifidobacterium probiotic foods or supplements as interventions to reduce the health risks among patients with MetS. More specifically, the authors used anthro- 


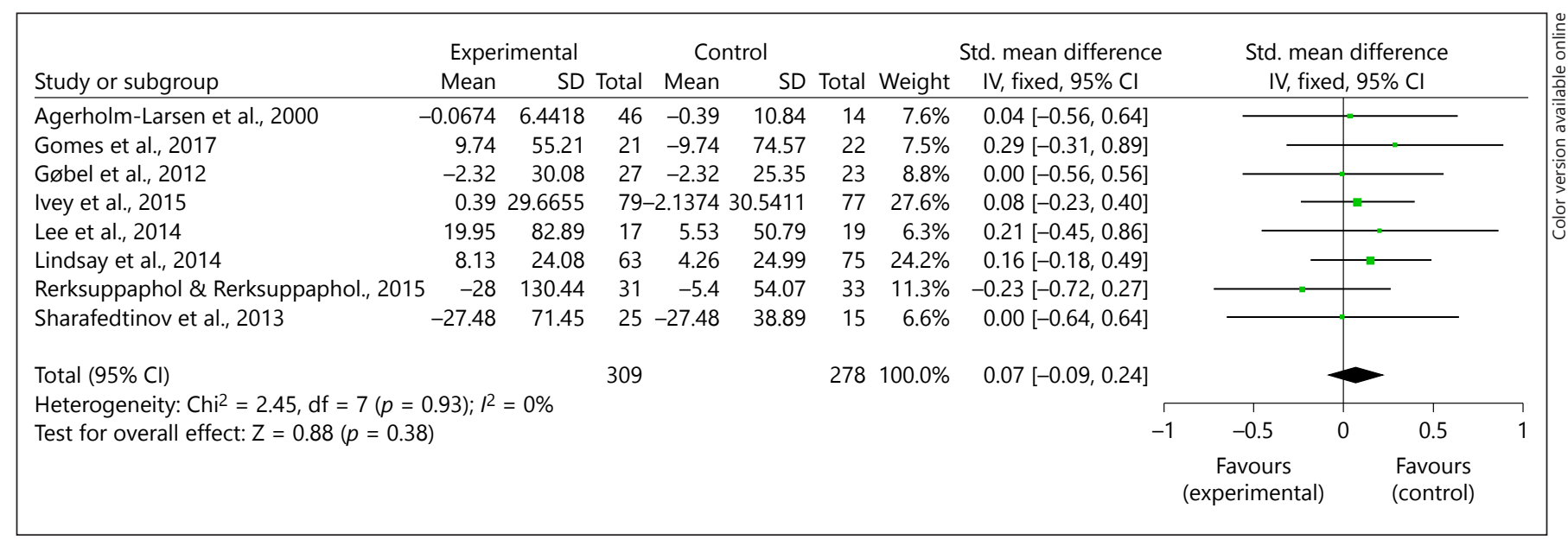

Fig. 15. Comparison of standardized mean net changes of triglycerides between probiotic groups and control groups. $I^{2}=0 \%, 95 \% \mathrm{CI}-0.09$ to $0.24, Z=0.88, p=0.38>0.05$. No significant difference in the comparison of standardized mean net changes of triglycerides.

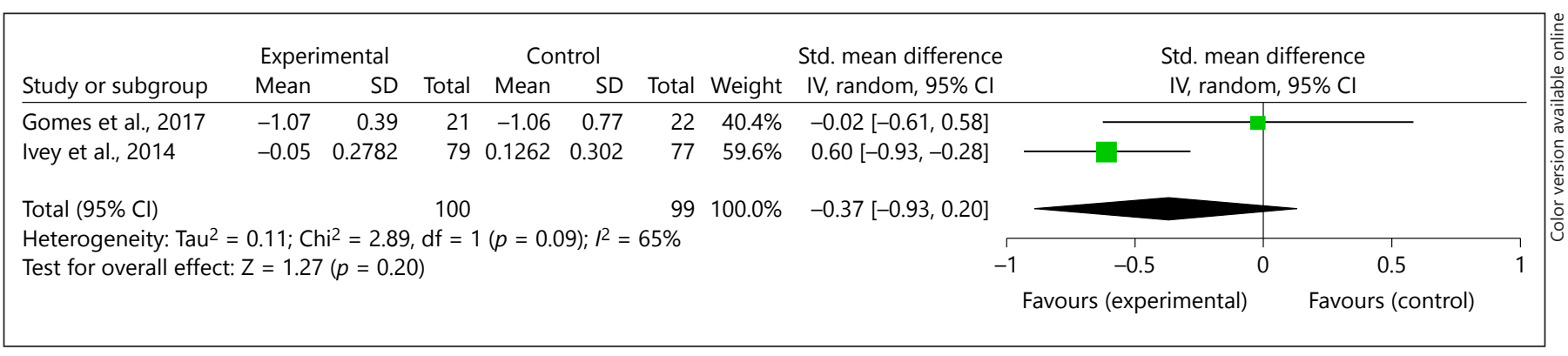

Fig. 16. Comparison of standardized mean net changes of HbAlc between probiotic groups and control groups. $\mathrm{Tau}^{2}=0.11, I^{2}=65 \%, 95 \%$ CI -0.93 to $0.20, Z=1.27, p=0.20$. No significant difference in the comparison of standardized mean net changes of HbAlc.

pometric and biochemical indicators as a measurement to evaluate the effectiveness of the intervention outcomes.

Primary mechanisms underlying the antagonistic effects of probiotics include improvement of the gut barrier function, increased competitive adherence to the mucosa and epithelium, gut microbiota modification, and regulation of the gut-associated lymphoid immune system. In this sense, probiotics communicate with the host through intestinal cell patterns recognition receptors, such as Toll-like receptors and nucleotide-binding oligomerization domain-containing protein-like receptors, which modulate important key signaling pathways, such as nuclear factor- $\kappa \mathrm{B}$ and mitogen-activated protein kinase, to enhance or suppress activation and influence downstream pathways $[68,69]$. Based on the results, the authors of this review concluded that Lactobacillus and
Bifidobacterium foods or supplements could be used as a possible intervention to bring specific beneficial changes for individuals with MetS. However, it is also important to point out that the probiotic intervention does little to nothing to improve blood pressure and lipid profiles, except for the LDL-C.

As suggested in the results section, when combining all the data from each RCT, there were significant differences in the following MetS indicators: LDL-C and BFP. However, researchers did not find significant differences in BFM, BMI, waist circumference, hip circumference, waist-to-hip ratio, fasting glucose, HbA1c, SBP, DBP, fasting insulin, TC, HDL-C, or triglycerides between the probiotics and placebo groups that were observed. Furthermore, the researchers found several RCTs studies with problems in research design and methodology. As explained in the Results section, all re- 
viewed studies were RCTs. Compared to other nonRCT research designs (e.g., observation studies), the RCTs design can provide more evidence-based analysis on the cause-and-effect relationships with minimal bias and confounding factors [70]. According to the Consolidated Standards of Reporting Trials statement [71], one critical part of reporting RCTs is to provide detailed information about the randomization process. Among the 18 reviewed RCTs studies, 12 of them provided explicit and replicable information about the method of randomization. The other 6 RCT studies have only mentioned that their studies were randomized in the methods section or elsewhere in the text or title $[49,51,53$, $55,61,64]$. Without detailed information about the randomization procedure, it became difficult for the reviewers to evaluate the quality of randomization (e.g., using pseudo-randomization such as time-period randomization).

In terms of sample size calculation, 8 RCT studies did not report any type of power calculations [42, 47, 49, 50, $52,54,55,61]$. One study reported post hoc calculation [66]. Five studies reported prior power calculation [43, $51,57,59,64]$. The other studies did not specify how they performed the power calculation. The inconsistency in providing sample size calculation should also be pointed out here. Furthermore, the study by Brahe et al. [38] only used a single-blind method, which may increase the likelihood of bias [72].

Another problem in the reviewed studies is that there was no consistency in selecting uniformed outcome measurement. As it was shown in Table 3, there were no anthropometric measurements in 3 studies [38, 43, 66]; meanwhile, 4 studies did not report any biochemical measurements $[49,51,52,54]$. The lack of consistency in reporting methods made it difficult for the reviewer to assess the overall effectiveness of using Lactobacillus and Bifidobacterium probiotic foods or supplements in MetS intervention.

In terms of food or medication interferences, Ivey et al. [64] suggested that some of their participants in both control and experimental groups used hypolipidemic medication or supplement; however, the researchers did not provide detailed information regarding the exact dosages of the medications or supplements. In addition, Lee et al. [55] suggested that they provided each participant in both control and experimental groups with equal dosage of Bofutsushosan over an 8-week period (3 g per administration, $15 \mathrm{~g}$ in total). No other study reported any abnormal usage of medication or supplement.

Probiotics and MetS, A Meta-Analysis

\section{Conclusion}

The results from this systematic review and meta-analysis indicated that probiotic food and supplements with Lactobacillus and Bifidobacterium genera have the potential to bring beneficial changes in BFP and LDL-C level for individuals with MetS. The results also suggested that probiotics did not have a significant effect in improving BFM, BMI, waist circumference, hip circumference, waist-to-hip ratio, fasting glucose, HbA1c, SBP, DBP, fasting insulin, TC, HDL-C, or triglycerides. Based on these 2 conclusions, the authors suggested that the probiotics should only be used as a complementary treatment to improve the overall effectiveness of other medical treatment.

In the future, researchers can improve the scientific rigor of their studies by using the triple-blind method, having large sample sizes with prior power calculation results, and providing complete reports on the randomizing process. Besides, it will be useful for future studies to provide more information regarding the sub-strains of the microbiome, as well as to provide detailed information about the participants. Such information will be useful for researchers to further analyze the data, and it will facilitate the designing of new intervention programs. Lastly, as it was mentioned in the previous chapter [30, 31 , the use of probiotics in treating MetS has been widely publicized on different media platforms; however, without further studies, several claims remain ambiguous. Thus, to provide accurate and responsible healthrelated information for the public and to better evaluate the efficacy of using probiotics in MetS treatment, researchers should also include more strains of probiotic bacteria in their future RCTs studies.

\section{Ethics Statement}

The authors have no ethical conflicts to disclose.

\section{Disclosure Statement}

The authors have no conflicts of interest to declare.

\section{Funding Sources}

This study was supported by a grant (No. 17050502200) from the Shanghai Municipal Science and Technology Commission, China. 


\section{Author Contributions}

Y.D. (corresponding author) contributed to the study conception and design, the acquisition of data, the development of the protocol, and the drafting of the manuscript. M.X. (equal contributor) contributed to the development of the protocol, the analysis and interpretation of the quantitative data, and the drafting of the manuscript. L.C. contributed to the development of the protocol and the critical revising of the final draft of the manuscript. A.B. contributed to the development of the protocol and the analysis and interpretation of the descriptive data.

\section{References}

1 Grundy SM. Metabolic syndrome scientific statement by the American Heart Association and the National Heart, Lung, and Blood Institute. Arterioscler Thromb Vasc Biol. 2005 Nov;25(11):2243-4.

2 Kaur J. A comprehensive review on metabolic syndrome. Cardiol Res Pract. 2014;2014: 943162.

3 Feldman R, Hegele R. Obesity/Metabolic Syndrome Overview. Comprehensive Medicinal Chemistry. 2007;II:381-7.

4 Reasner CA. Reducing cardiovascular complications of type 2 diabetes by targeting multiple risk factors. J Cardiovasc Pharmacol. 2008 Aug;52(2):136-44.

5 World Health Organization. (2018, February 16). Obesity and overweight. Retrieved from: http://www.who.int/news-room/fact-sheets/ detail/obesity-and-overweight.

6 Hales CM, Carroll MD, Fryar CD, Ogden CL; National Center for Health Statistics. (2017, October 13). Prevalence of Obesity among Adults and Youth: United States, 2015-2016. Retrieved from: https://www.cdc.gov/nchs/ data/databriefs/db288.pdf.

7 NHLBI Obesity Education Initiative Expert Panel on the Identification, Evaluation, and Treatment of Obesity in Adults (US), 1998. Clinical guidelines on the identification, evaluation, and treatment of overweight and obesity in adults: The evidence report. Bethesda, MD: National Heart, Lung, and Blood Institute in cooperation with the National Institute of Diabetes and Digestive and Kidney Diseases.

8 Murphy SL, Xu J, Kochanek KD, Curtin SC, Arias E; Division of Vital Statistics. (2017, November 27). National Vital Statistics Reports: Deaths: Final Data for 2015. Retrieved from: https://www.cdc.gov/nchs/data/nvsr/nvsr66/ nvsr66_06.pdf.

9 Moy FM, Bulgiba A. High prevalence of vitamin D insufficiency and its association with obesity and metabolic syndrome among Malay adults in Kuala Lumpur, Malaysia. BMC Public Health. 2011 Sep;11(1):735.

10 Sender R, Fuchs S, Milo R. Revised Estimates for the Number of Human and Bacteria Cells in the Body. PLoS Biol. 2016 Aug; 14(8): e1002533.

11 Bull MJ, Plummer NT. Part 1: The Human Gut Microbiome in Health and Disease. Integr Med (Encinitas). 2014 Dec;13(6):17-22.

$12 \mathrm{Wu} \mathrm{HJ}, \mathrm{Wu}$. The role of gut microbiota in immune homeostasis and autoimmunity. Gut Microbes. 2012 Jan-Feb;3(1):4-14.
13 Tlaskalová-Hogenová H, Stěpánková $\mathrm{R}$, Kozáková H, Hudcovic T, Vannucci L, Tučková $\mathrm{L}$, et al. The role of gut microbiota (commensal bacteria) and the mucosal barrier in the pathogenesis of inflammatory and autoimmune diseases and cancer: contribution of germ-free and gnotobiotic animal models of human diseases. Cell Mol Immunol. 2011 Mar;8(2):110-20.

14 Yu CG, Huang Q. Recent progress on the role of gut microbiota in the pathogenesis of inflammatory bowel disease. J Dig Dis. 2013 Oct;14(10):513-7.

15 Loh G, Blaut M. Role of commensal gut bacteria in inflammatory bowel diseases. Gut Microbes. 2012 Nov-Dec;3(6):544-55.

16 Guinane CM, Cotter PD. Role of the gut microbiota in health and chronic gastrointestinal disease: understanding a hidden metabolic organ. Therap Adv Gastroenterol. 2013 Jul; 6(4):295-308

17 Parnell JA, Reimer RA. Prebiotic fiber modulation of the gut microbiota improves risk factors for obesity and the metabolic syndrome. Gut Microbes. 2012 Jan-Feb;3(1):29-34

18 Tilg H, Moschen AR. Food, immunity, and the microbiome. Gastroenterology. 2015 May;148(6):1107-19.

19 Conlon MA, Bird AR. The impact of diet and lifestyle on gut microbiota and human health. Nutrients. 2014 Dec;7(1):17-44.

20 Festi D, Schiumerini R, Eusebi LH, Marasco G, Taddia M, Colecchia A. Gut microbiota and metabolic syndrome. World J Gastroenterol. 2014 Nov;20(43):16079-94.

21 Grover S, Malhotra N, Chakrabarti S, Kulhara P. Metabolic syndrome in bipolar disorders. Indian J Psychol Med. 2012 Apr;34(2):110-8.

22 Carding S, Verbeke K, Vipond DT, Corfe BM, Owen LJ. Dysbiosis of the gut microbiota in disease. Microb Ecol Health Dis. 2015 Feb; 26(0):26191.

23 Le Barz M, Anhê FF, Varin TV, Desjardins Y, Levy E, Roy D, et al. Probiotics as Complementary Treatment for Metabolic Disorders. Diabetes Metab J. 2015 Aug;39(4):291-303.

24 Fijan S. Microorganisms with claimed probiotic properties: an overview of recent literature. Int J Environ Res Public Health. 2014 May;11(5):4745-67.

25 Pithva S, Ambalam PM, Rajiv B. Potential of Probiotic Lactobacillus Strains as Food Additives. Food Additive; 2012.

26 Charteris WP, Kelly PM, Morelli L, Collins JK. Antibiotic susceptibility of potentially probiotic Lactobacillus species. J Food Prot. 1998 Dec;61(12):1636-43.

27 Didari T, Solki S, Mozaffari S, Nikfar S, Abdollahi M. A systematic review of the safety of probiotics. Expert Opin Drug Saf. 2014 Feb; 13(2):227-39.

28 Hemarajata P, Versalovic J. Effects of probiotics on gut microbiota: mechanisms of intestinal immunomodulation and neuromodulation. Therap Adv Gastroenterol. 2013 Jan; 6(1):39-51.

29 Swidsinski A, Loening-Baucke V, Lochs $\mathrm{H}$, Hale LP. Spatial organization of bacterial flora in normal and inflamed intestine: a fluorescenceinsituhybridization studyinmice. World J Gastroenterol. 2005 Feb;11(8):1131-40.

30 Bonevski B, Wilson A, Henry DA. An analysis of news media coverage of complementary and alternative medicine. PLoS One. 2008 Jun;3(6):e2406

31 Brinich MA, Mercer MB, Sharp RR. An analysis of online messages about probiotics. BMC Gastroenterol. 2013 Jan;13(1):5.

32 Moher D, Shamseer L, Clarke M, Ghersi D, Liberati A, Petticrew M, et al.; PRISMA-P Group. Preferred reporting items for systematic review and meta-analysis protocols (PRISMA-P) 2015 statement. Syst Rev. 2015 Jan; $4(1): 1$.

33 Higgins JP, Green S. Cochrane handbook for systematic reviews of interventions. Chisester. Wiley-Blackwell; 2017.

34 Higgins JP, Thompson SG, Deeks JJ, Altman DG. Measuring inconsistency in meta-analyses. BMJ. 2003 Sep;327(7414):557-60.

35 Schroll JB, Moustgaard R, Gøtzsche PC. Dealing with substantial heterogeneity in Cochrane reviews. Cross-sectional study. BMC Med Res Methodol. 2011 Feb;11(1):22.

36 Review Manager (RevMan) [Computer program]. Version 5.3. Copenhagen: The Nordic Cochrane Centre, the Cochrane Collaboration, 2014. (Version 5.3.) [Computer software]. (n.d.). Retrieved from: https://community.cochrane.org/help/tools-and-software/ revman-5.

37 Kemp R, Prasad V. Surrogate endpoints in oncology: when are they acceptable for regulatory and clinical decisions, and are they currently overused? BMC Med. 2017 Jul;15(1):134.

38 Brahe LK, Le Chatelier E, Prifti E, Pons N, Kennedy S, Blædel T, et al. Dietary modulation of the gut microbiota-a randomised controlled trial in obese postmenopausal women. Br J Nutr. 2015 Aug;114(3):406-17. 
39 Cohen DR, Todd S, Gregory WM, Brown JM. Adding a treatment arm to an ongoing clinical trial: a review of methodology and practice. Trials. 2015 Apr;16(1):179.

40 O'Neil M, Berkman N, Hartling L, Chang S, Anderson J, Motu'apuaka M, et al. Observational evidence and strength of evidence domains: case examples. Syst Rev. 2014 Apr; 3(1):35.

41 OCEBM Levels of Evidence Working Group* "The Oxford 2011 Levels of Evidence". Retrieved from: https://www.cebm.net/wp-content/uploads/2014/06/CEBM-Levels-of-Evidence-2.1.pdf.

42 Sharafedtinov KK, Plotnikova OA, Alexeeva RI, Sentsova TB, Songisepp E, Stsepetova J, et al. Hypocaloric diet supplemented with probiotic cheese improves body mass index and blood pressure indices of obese hypertensive patients - a randomized double-blind placebo-controlled pilot study. Nutr J. 2013 Oct; 12(1): 138

43 Lindsay KL, Kennelly M, Culliton M, Smith T, Maguire OC, Shanahan F, et al. Probiotics in obese pregnancy do not reduce maternal fasting glucose: a double-blind, placebo-controlled, randomized trial (Probiotics in Pregnancy Study). Am J Clin Nutr. 2014 Jun;99(6): 1432-9.

44 Sagun G, Oguz A, Karagoz E, Filizer AT, Tamer G, Mesci B. Application of alternative anthropometric measurements to predict metabolic syndrome. Clinics (São Paulo). 2014;69(5):347-53.

45 Mooney SJ, Baecker A, Rundle AG. Comparison of anthropometric and body composition measures as predictors of components of the metabolic syndrome in a clinical setting. Obes Res Clin Pract. 2013 Jan-Feb;7(1):e5566.

46 Weisell RC. Body mass index as an indicator of obesity. Asia Pac J Clin Nutr. 2002;11(s8 S8):S681-4.

47 Chung HJ, Yu JG, Lee IA, Liu MJ, Shen YF, Sharma SP, et al. Intestinal removal of free fatty acids from hosts by Lactobacilli for the treatment of obesity. FEBS Open Bio. 2016 Jan;6(1):64-76.

48 Rerksuppaphol S, Rerksuppaphol L. A Randomized Double-blind Controlled Trial of Lactobacillus acidophilus Plus Bifidobacterium bifidum versus Placebo in Patients with Hypercholesterolemia. J Clin Diagn Res. 2015 Mar;9(3):KC01-04.

49 Vajro P, Mandato C, Licenziati MR, Franzese A, Vitale DF, Lenta S, et al. Effects of Lactobacillus rhamnosus strain GG in pediatric obesity-related liver disease. J Pediatr Gastroenterol Nutr. 2011 Jun;52(6):740-3.

50 Gøbel RJ, Jensen SM, Frøkiaer H, Mølgaard C, Michaelsen KF. Obesity, inflammation and metabolic syndrome in Danish adolescents. Acta Paediatr. 2012 Feb;101(2):192-200.

51 Kadooka Y, Sato M, Ogawa A, Miyoshi M, Uenishi H, Ogawa H, et al. Effect of Lactobacillus gasseri SBT2055 in fermented milk on abdominal adiposity in adults in a randomised controlled trial. Br J Nutr. 2013 Nov; 110(9):1696-703

52 Zarrati M, Shidfar F, Nourijelyani K, Mofid V, Hossein zadeh-Attar MJ, Bidad K, et al. Lactobacillus acidophilus La5, Bifidobacterium BB12, and Lactobacillus casei DN001 modulate gene expression of subset specific transcription factors and cytokines in peripheral blood mononuclear cells of obese and overweight people. Biofactors. 2013 Nov-Dec; 39(6):633-43.

53 Kadooka Y, Sato M, Imaizumi K, Ogawa A, Ikuyama K, Akai Y, et al. Regulation of abdominal adiposity by probiotics (Lactobacillus gasseri SBT2055) in adults with obese tendencies in a randomized controlled trial. Eur J Clin Nutr. 2010 Jun;64(6):636-43.

54 Hariri M, Salehi R, Feizi A, Mirlohi M, Ghiasvand $\mathrm{R}$, Habibi N. A randomized, doubleblind, placebo-controlled, clinical trial on probiotic soy milk and soy milk: effects on epigenetics and oxidative stress in patients with type II diabetes. Genes Nutr. 2015 Nov; 10(6):52.

55 Lee SJ, Bose S, Seo JG, Chung WS, Lim CY, Kim H. The effects of co-administration of probiotics with herbal medicine on obesity, metabolic endotoxemia and dysbiosis: a randomized double-blind controlled clinical trial. Clin Nutr. 2014 Dec;33(6):973-81.

56 Gomes AC, de Sousa RG, Botelho PB, Gomes TL, Prada PO, Mota JF. The additional effects of a probiotic mix on abdominal adiposity and antioxidant Status: A double-blind, randomized trial. Obesity (Silver Spring). 2017 Jan;25(1):30-8.

57 Sanchez M, Darimont C, Drapeau V, Emady-Azar S, Philippe L, Ammon-Zuffrey C, et al. Effect of Lactobacillus Rhamnosus CGMCC1.3724 Supplementation on Weight loss and Maintenance in Obese Men and Women. Br J Nutr. 2014 Apr 28;111(8): 1507-19.

58 Janssen I, Katzmarzyk PT, Ross R. Waist circumference and not body mass index explains obesity-related health risk. Am J Clin Nutr. 2004 Mar;79(3):379-84.

59 Ilmonen J, Isolauri E, Poussa T, Laitinen K. Impact of dietary counselling and probiotic intervention on maternal anthropometric measurements during and after pregnancy: a randomized placebo-controlled trial. Clin Nutr. 2011 Apr;30(2):156-64.

60 Mirmiran P, Mohammadi F, Sarbazi N, Allahverdian S, Azizi F. Gender differences in dietary intakes, anthropometrical measurements and biochemical indices in an urban adult population: the Tehran Lipid and Glucose Study. Nutr Metab Cardiovasc Dis. 2003 Apr;13(2):64-71.

61 Agerholm-Larsen L, Raben A, Haulrik N, Hansen AS, Manders M, Astrup A. Effect of 8 week intake of probiotic milk products on risk factors for cardiovascular diseases. Eur J Clin Nutr. 2000 Apr;54(4):288-97.

62 Bøg-Hansen E, Lindblad U, Gullberg B, Melander A, Råstam L. Metabolic disorders associated with uncontrolled hypertension. Diabetes Obes Metab. 2003 Nov;5(6):379-87.

63 Kimm H, Mok Y, Lee SJ, Lee S, Back JH, Jee $\mathrm{SH}$. The J-curve between Diastolic Blood Pressure and Risk of All-cause and Cardiovascular Death. Korean Circ J. 2018 Jan;48(1): 36-47.

64 Ivey KL, Hodgson JM, Kerr DA, Thompson PL, Stojceski B, Prince RL. The effect of yoghurt and its probiotics on blood pressure and serum lipid profile; a randomised controlled trial. Nutr Metab Cardiovasc Dis. 2015 Jan; 25(1):46-51.

65 Eckel RH, Grundy SM, Zimmet PZ. The metabolic syndrome. Lancet. 2005 Apr;365(9468): 1415-28.

66 Ivey KL, Hodgson JM, Kerr DA, Lewis JR, Thompson PL, Prince RL. The effects of probiotic bacteria on glycaemic control in overweight men and women: a randomised controlled trial. Eur J Clin Nutr. 2014 Apr;68(4): $447-52$.

67 Johnson JL, Duick DS, Chui MA, Aldasouqi SA. Identifying prediabetes using fasting insulin levels. Endocr Pract. 2010 Jan-Feb; 16(1):47-52.

68 World Health Organization, Food and Agricultural Organization of the United Nations. Health and Nutritional Properties of Probiotics in Food including Powder Milk with Live Lactic Acid Bacteria. FAO Nutrition Paper. 2001;85:1-33.

69 Fontana L, Bermudez-Brito M, Plaza-Diaz J, Muñoz-Quezada S, Gil A. Sources, isolation, characterisation and evaluation of probiotics. Br J Nutr. 2013 Jan;109(S2 Suppl 2):S35-50.

70 Spieth PM, Kubasch AS, Penzlin AI, Illigens BM, Barlinn K, Siepmann T. Randomized controlled trials 499028 a matter of design. Neuropsychiatr Dis Treat. 2016 Jun;12:13419.

71 Schulz KF, Altman DG, Moher D; CONSORT Group. CONSORT 2010 statement: updated guidelines for reporting parallel group randomised trials. BMJ. 2010 Mar;340 mar23 1:c332.

72 Page SJ, Persch AC. Recruitment, retention, and blinding in clinical trials. Am J Occup Ther. 2013 Mar-Apr;67(2):154-61. 